\title{
Linx
}

Revue des linguistes de l'université Paris X Nanterre

$12 \mid 2002$

"Comme la lettre dit la vie »

\section{« Francien » et « français de Paris »}

\section{Anthony Lodge}

\section{OpenEdition}

\section{Journals}

Édition électronique

URL : http://journals.openedition.org/linx/1296

DOI : 10.4000/linx.1296

ISSN : 2118-9692

\section{Éditeur}

Presses universitaires de Paris Nanterre

\section{Édition imprimée}

Date de publication : 1 octobre 2002

Pagination : 149-172

ISSN : 0246-8743

\section{Référence électronique}

Anthony Lodge, « «Francien » et «français de Paris » », Linx [En ligne], 12 | 2002, mis en ligne le 10 octobre 2012, consulté le 19 avril 2019. URL : http://journals.openedition.org/linx/1296 ; DOI :

10.4000/linx.1296

Ce document a été généré automatiquement le 19 avril 2019.

Département de Sciences du langage, Université Paris Ouest 


\title{
« Francien » et « français de Paris »
}

\author{
Anthony Lodge
}

\section{Introduction}

Dans l'histoire des langues standard, les sources de la standardisation constituent, cela va de soi, un domaine de recherche privilégié, et l'on conçoit sans difficulté l'importance qu'a toujours eu ce sujet pour les historiens du français. Cela n'a pas toujours suffi, néanmoins, à garantir la productivité des débats en ce domaine : la proximité des notions de «langue standard» et de "nation» dans l'esprit des observateurs a bien souvent empêché que la question des origines de ce que l'on appelle souvent «la langue » tout court reste à l'abri d'a priori idéologiques concernant «la genèse d'une nation ». On pourrait citer en exemple le débat parfois bruyant qui a eu lieu dans les années 80 et 90 sur l'emploi de l'étiquette francien. L'idée, née au XIX ${ }^{e}$ siècle et retenue par de nombreux historiens du français tout au long du XXe, que la langue standard (le «francais ») n'est que le prolongement historique $\mathrm{du}$ dialecte de l'Ile-de-France (le "francien»), fut vigoureusement contestée dans les années 80 . Elle fut remplacée par une nouvelle orthodoxie qui vit émerger le français standard au Moyen Age d'une manière moins centraliste et plus consensuelle à partir d'une langue écrite supra-régionale, constituée à date ancienne par une sélection (pour certains, consciente et politiquement motivée) d'éléments tirés de plusieurs dialectes.

2 Dans cet article, dédié à l'une des grandes spécialistes du français sous tous ses aspects, nous entendons reprendre la question épineuse des débuts de la standardisation en français, en passant en revue, dans un premier temps, les tentatives d'explication présentées jusqu'ici, et en ébauchant ensuite une approche qui cadrera peut-être mieux avec les tendances actuelles de la dialectologie historique, et qui évitera en tous cas d'attribuer à nos ancêtres des rapprochements téléologiques entre "langue» et «nation». 


\section{La question du francien}

3 Au cours des XII ${ }^{e}$-XIII e siècles, il allait de soi que l'Ile-de-France (la "France» dans la terminologie de l'époque) possédait, comme tout autre lieu, sa propre variété de parler régional. Roger Bacon a distingué dans le gallo-roman septentrional quatre zones dialectales :

Nam et idiomata ejusdem linguae variantur apud diversos, sicut patet de lingua gallicana, quae apud Gallicos et Picardos et Normannos et Burgundos et caeteros multiplici idiomate variatur. Et quod proprie et intelligentibiliter dicitur in idiomate Picardorum horrescit apud Burgundos, immo apud Gallicos viciniores quanto magis igitur accidet hoc apud linguas diversas?

(Opus Majus, II, 80-1)

4 A côté du terme latin gallicum, le terme français désignant le parler de l'Ile-de-France était bien entendu le françois (voir Bader 1969: 72-87, Walter 1988: 83 et Fondet 1980), mais très tôt cette étiquette a été appliquée selon une synecdoque à la langue française en général (sur ce procédé voir Joseph 1987: 2). La philologie romane du XIX ${ }^{e}$ siècle, en quête d'un étiquetage plus précis des variétés de langue, a cherché à éliminer la confusion entre le nom du dialecte de l'Ile-de-France (le françois) et celui de la future langue standard (le français). En Allemagne H. Suchier avait proposé de distinguer entre Francisch et Französisch, et un peu plus tard, en France, Gaston Paris (1889: 475) créa un nouveau terme, le francien, pour remplacer le françois médiéval. La formulation des lois phonétiques rendait nécessaire une succession ininterrompue d'états dialectaux purs entre le latin et le français moderne, et il est légitime de penser que, en créant ce terme, G. Paris cherchait non seulement à instaurer une certaine transparence terminologique, mais aussi à doter la langue standard naissante des qualités historiques qu'on pensait alors indispensables, c'est-à-dire une localisation centrale à proximité de la capitale et un dialecte-source "pur» (en l'occurrence à l'abri de contaminations germaniques). Il est significatif que Ferdinand Brunot ait insisté lui aussi sur la pureté de ce dialecte: «le francien ne doit pas être considéré comme un amalgame » (Brunot 1905 : 325).

5 Tout au long du $\mathrm{XX}^{\mathrm{e}}$ siècle, le terme francien, bien qu'évité par la plupart des dialectologues, a été largement utilisé dans les manuels d'histoire de la langue (voir Pope 1935 : 33, François 1959 : 92, von Wartburg 1962 : 90, Bec 1971 : 6, Cohen 1987 : 86, Rickard 1989 : 41). Suivant la typologie établie par Haugen (1966) des processus entrant en jeu dans la standardisation des langues, le dialecte de l'Ile-de-France (le francien) aurait été " sélectionné » au XII ${ }^{e}$ siècle, « élaboré » au cours du bas Moyen Age, « codifié » aux XVII -XVIII ${ }^{e}$ siècles, et "accepté" par l'ensemble de la population devenue maintenant " française ", au cours des XIX ${ }^{\mathrm{e}}-\mathrm{XX}$ e siècles. Dans le courant des années 80 , cependant, l'étiquette francien fit l'objet de critiques sévères. Chaurand (1983: 91), par exemple, considère que le sens de ce terme est circulaire, et il signale à juste titre qu'il n'existe aucun texte écrit attestant la nature de ce dialecte avant l'émergence d'une langue standard embryonnaire au XIII ${ }^{e}$ siècle, dont le francien était censé être l'antécédent. Ceci l'a conduit à écarter le dialecte médiéval de l'Ile-de-France comme étant une "variété hybride et peu distincte, dont nous ne savons rien de sûr avant le XIII ${ }^{\mathrm{e}}$ siècle ».

6 Bergounioux (1989) va plus loin: il soutient qu'un dialecte rural nécessitant l'étiquette francien n'a jamais existé en Ile-de-France, persuadé, semble-t-il, que celui-ci avait été anéanti dès sa naissance par l'influence linguistique de la grande ville (mais il n'indique 
pas les origines du parler de cette ville). Tout en niant l'existence de traces de ce dialecte dans les textes médiévaux, Bergounioux élimine aussi l'incidence des nombreux documents sur le parler rural de cette région aux XVII ${ }^{\mathrm{e}}-\mathrm{XVIII}{ }^{\mathrm{e}}$ siècles. Il va jusqu'à dénigrer même les attestations modernes de ce dialecte. Pour lui, le francien est une expression sans référent, un terme à motivation exclusivement politique, créé par Gaston Paris dans le but de donner une légitimation historique à la langue standard que la Troisième République cherchait alors à imposer sur l'ensemble de l'Hexagone.

7 Cerquiglini (1993 : 118) reprend cet argument pour consolider son hypothèse d'une langue standard fondée sur l'écrit: pour lui, on peut se passer du concept de francien, puisque le français standard, que l'on croyait dérivé du francien, descend en fait d'une variété écrite composite, constituée aux $\mathrm{X}^{\mathrm{e}}-\mathrm{XI}^{\mathrm{e}}$ siècles par une coterie de scribes et d'auteurs littéraires ayant rassemblé des éléments linguistiques de plusieurs dialectes. N'ayant aucunement besoin d'un dialecte parlé de base puisant ses origines dans la région parisienne, Cerquiglini est amené, comme Bergounioux, à affirmer que Paris et l'Ile-deFrance n'avaient pas de dialecte du tout:

L'Ile-de-France ne se distinguait par aucun dialecte. Jusqu'aux portes, et sans doute dans les rues de la modeste bourgade parisienne, on devait parler picard, normand ou orléanais.

8 Décrire la plus grande ville de l'Occident comme une "modeste bourgade » pourrait paraître étrange, mais tout dépend évidemment de l'époque où l'on se situe. On remarquera néanmoins que cette affirmation fragilise du même coup la pensée de Bergounioux pour qui le dialecte rural de l'Ile-de-France avait très tôt disparu sous l'influence linguistique de la grande ville. Plus gênante est la suggestion que, tandis que d'autres régions possédaient leur propre dialecte, ceci ne valait pas pour l'Ile-de-France : prétendre qu'une partie d'une communauté linguistique puisse être dépourvue de dialecte, c'est donner au mot « dialecte » un sens que peu de dialectologues pourraient de nos jours reconnaître. L'approche de Cerquiglini ressemble beaucoup à celle de l'idéologie normative, qui dépeint la langue standard comme une norme non localisée, non rattachée à une région particulière, sans pourtant être une variété hybride, et comme une langue pourvue de toutes les caractéristiques de l'urbanité, sans pourtant être urbaine (voir Mugglestone 1995 : 26-34).

9 La notion de «dialecte » qui semble dominer ici se rapproche du mythique « dialecte pur» de la dialectologie traditionnelle : rustique, homogène, nettement délimité sur le plan de l'espace. Seules les variétés de langue munies de ces traits peuvent revendiquer véritablement le titre de « dialecte ». Comme il est impossible de démontrer que le parler de l'Ile-de-France les possédait, celui-ci ne pouvait être un dialecte. Ergo, le francien n'a pas existé. Appliquer cette notion de « dialecte » à la situation linguistique en Picardie, en Normandie et dans le Northumberland aurait soulevé, bien entendu, des difficultés identiques. Si la région parisienne avait, au XII ${ }^{\mathrm{e}}$ siècle, une population - et nous savons que c'était une des régions les plus peuplées de France (voir Fourquin 1956) - elle possédait nécessairement un dialecte. Selon toute probabilité, ce dialecte n'était pas homogène et se fondait imperceptiblement dans les dialectes des régions environnantes, mais il en allait de même pour tous les autres dialectes du gallo-roman. Que l'on appelle ou non ce dialecte le francien a très peu d'importance.

10 Il apparaitt ainsi que les arguments des opposants à la thèse francienne soulèvent presque autant de problèmes qu'ils n'en résolvent, mais il serait abusif de penser que leur apport soit surtout négatif. Ils nous obligent en tout état de cause aux observations suivantes : (a) 
la base empirique sur laquelle repose la représentation du dialecte francien dans les manuels d'usage est extrêmement fragile ; et (b) le français standard est à l'origine une variété composite, comportant un grand nombre d'éléments phonologiques et morphologiques qui n'étaient pas indigènes en Ile-de-France. Ces deux points sont difficiles à démêler, et posent le problème de savoir jusqu'où on peut reconstituer la situation dialectale de la moitié nord de la France au Moyen Age (voir Dees 1988, 1989).

\section{La reconstruction du « francien » médiéval}

Comme l'a montré Jacques Chaurand, reconstruire le parler de l'Ile-de-France à l'époque médiévale sans tomber dans la circularité est exceptionnellement difficile. Dans les manuels d'ancien français, le francien est rarement décrit de manière empirique. Son existence comme antécédent (hypothétique) de la langue standard est donnée a priori : il s'agit du dialecte non marqué en face duquel les autres peuvent être définis, le dialecte dont la qualité essentielle est de ne posséder aucun des traits régionaux des autres dialectes de l'ancien français. En dehors d'indices toponymiques, aucun document écrit remontant au-delà du XIII ${ }^{e}$ siècle et attestant sans ambiguïté les formes locales n'est parvenu jusqu'à nous. De plus, les vernaculaires ruraux traditionnels, qui ailleurs en France sont le prolongement moderne des dialectes médiévaux, ont été effacés en région parisienne à l'époque industrielle (XIX'-XXe siècles) de manière beaucoup plus efficace. Le rapport symbiotique entre la ville et son arrière-pays a fait que, dès le Moyen Age, le francien a été soumis à une influence de la ville plus profonde que les dialectes situés dans des régions plus éloignées (voir Dauzat 1922: 53 et 139, carte VI). Cela dit, nos sources en ce qui concerne le francien ne sont pas aussi lacunaires que tout cela pourrait le laisser croire.

12 S'agissant de sources médiévales, l'Ile-de-France est aussi bien dotée de documents d'archives que les autres régions du nord de la France. Beaucoup d'entre eux furent admirablement décrits il y a plus d'un siècle (voir Matzke 1880, 1881). A ces documents on peut, bien entendu, ajouter les rôles de tailles parisiens étudiés par Michaëlsson (voir en particulier Michaëlsson 1959). Pour ce qui est des documents remontant au début de l'époque moderne, il est évident qu'ils sont plus nombreux que dans d'autres régions de France (voir Simoni-Aurembou 1973b). Tout en acceptant comme principe que les données linguistiques historiques sont toujours mauvaises, il n'en reste pas moins qu'elles sont moins mauvaises en Ile-de-France qu'elles pourraient l'être. Il existe par ailleurs une autre approche de la reconstruction historique des langues qu'il serait utile d'explorer. Comme le dit William Labov (1994: 27) :

The close examination of the present shows that much of the past is still with us. The study of history benefits from the continuity of the past as well as from analogies with the present.

Il convient naturellement d'appliquer ce principe avec précaution: nous ne disposons d'aucune clef qui nous permette de distinguer de manière infaillible entre les traits présents dans le monde moderne qui sont là depuis longtemps et ceux qui sont apparus depuis peu. Cela dit, il est possible que les traces de configurations dialectales d'époques antérieures soient encore visibles dans les atlas linguistiques constitués si minutieusement par les dialectologues au cours du $\mathrm{XX}^{\mathrm{e}}$ siècle. L'emploi judicieux des données cartographiques et des renseignements provenant de sources écrites du début de 
l'époque moderne nous permettra peut-être d'en savoir un peu plus sur l'histoire du parler de l'Ile-de-France ainsi que sur ses rapports avec la langue standard.

Or, si nous interrogeons la distribution de variables spatiales dans les vernaculaires ruraux traditionnels du nord gallo-roman telle qu'elle apparaît dans le monumental Atlas Linguistique de la France de Gilliéron et Edmont (1901-11), elle est loin d'être muette sur les états passés de la langue. Dans ce qui suit nous ne chercherons pas à circonscrire en termes linguistiques le dialecte médiéval de l'Ile-de-France. Nous nous bornerons à faire des constatations très simples, inspirées par la remarquable étude de Claire Fondet (1995) concernant la distribution de certaines isoglosses dans l'ALF dans la zone linguistique située dans un rayon de $100 \mathrm{~km}$ autour de Paris (voir aussi Gauthier 1995 et SimoniAurembou 1995). Cette étude a déjà retenu l'attention de Picoche et Marchello-Nizia (1994: 22), et les conclusions qu'elles en tirent ne diffèrent pas très sensiblement des nôtres. Nous constatons d'abord un groupe de traits phonétiques et morphologiques affectant un grand nombre de mots, qui se retrouvent sur l'ensemble de la zone et qui distinguent le parler de cette zone de ceux qui se trouvent respectivement au nord, au sud, à l'est et à l'ouest; ensuite, nous discernons un autre groupe de traits-clef qui pénètrent cette zone de l'extérieur et qui détruisent l'apparente homogénéité du dialecte.

Examinons dans un premier temps les isoglosses visibles dans l'ALF qui, prises ensemble, forment une sorte de cadre autour de l'Ile-de-France et de l'Orléanais. Il s'agit de la distribution de quatre isoglosses importantes formées par des traits partagés grosso modo par toutes les variétés situées à l'intérieur de ce cadre, ce qui les distingue de variétés situées en dehors.

16 (A) $\mathrm{Au}$ long du côté septentrional du cadre,nous trouvons la limite sud de la palatalisation de [ka-]. Il s'agit de la célèbre Ligne Joret : 


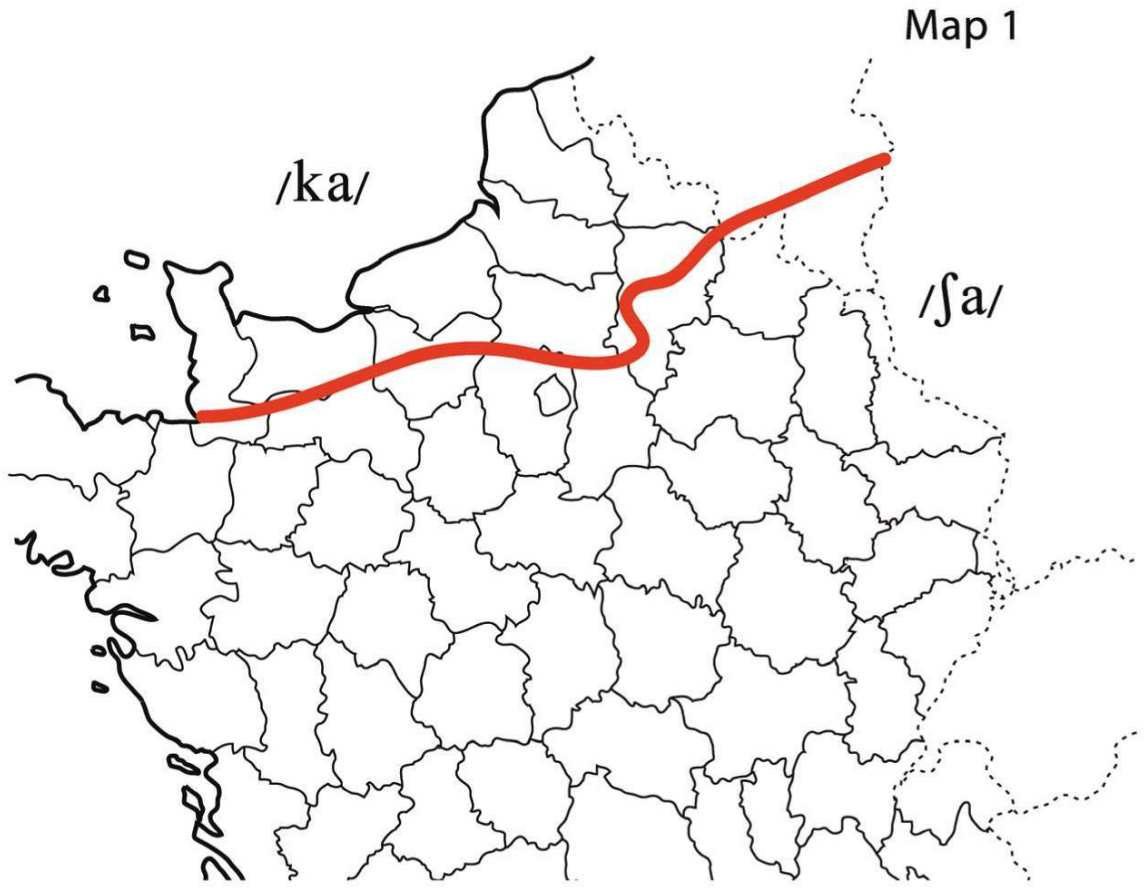

17 En Picardie et dans la partie nord de la Normandie, le [ka] latin demeure non palatalisé, tandis qu'ailleurs dans le domaine d'oïl il se palatalise $>[\mathrm{Ja} / \mathrm{Se} / \mathrm{t}]]$. La

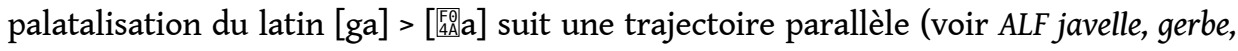
jardin), ainsi que l'isoglosse de la non-palatalisation du groupe [ke- / ki-] : voir ALF 704, ici. On trouvera d'autres exemples à : ciboule (ALF 284), cendre (ALF 210), racine (ALF 1126), ronce (ALF 1163). En Picardie et dans la partie nord de la Normandie, le [ke/i] latin passe à [Si / $\mathrm{e}$ ] alvéo-palatal, tandis que dans le reste de la langue d'oïl, il passe à [si/se] alvéolaire.

18 (B) Au long du côté occidental du cadre nous trouvons la limite de la différentiation de la diphtongue [ow] sortie de [o] accentué, différenciée en [ew] : 


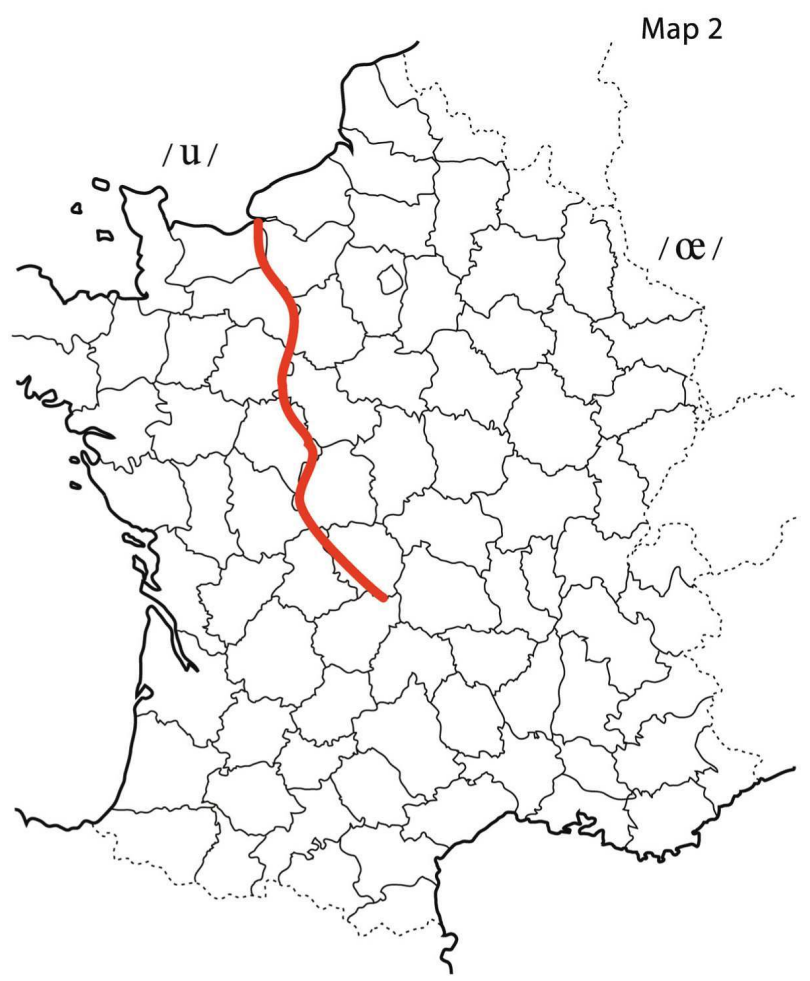

On trouvera d'autres exemples à : noeud (ALF 915) et dans Gauthier (1995 : 31). Il semblerait que le [o] latin accentué s'était diphtongué en > [ow] sur toute l'étendue de la langue d'oil avant le XII ${ }^{\mathrm{e}}$ siècle (voir Chauveau 1989 : 145-191). Tandis que dans l'ouest, cette diphtongue s'est nivelée ensuite en > [u], plus à l'est, elle s'est différenciée en [ew], se nivelant plus tard en > [oe]. Cette différentiation aurait débuté dans le nord du domaine d'oïl. A partir du XVI ${ }^{\mathrm{e}}$ siècle, la prononciation en [oe] a commencé à se diffuser à partir de Paris, vers l'ouest de la France (voir Wüest 1979b : 204-7 ; Pfister 1993 : 30-31). (C) Au long du côté oriental du cadre, nous trouvons la limite des consonnes épenthétiques dans le groupe [n_r], etc. : 


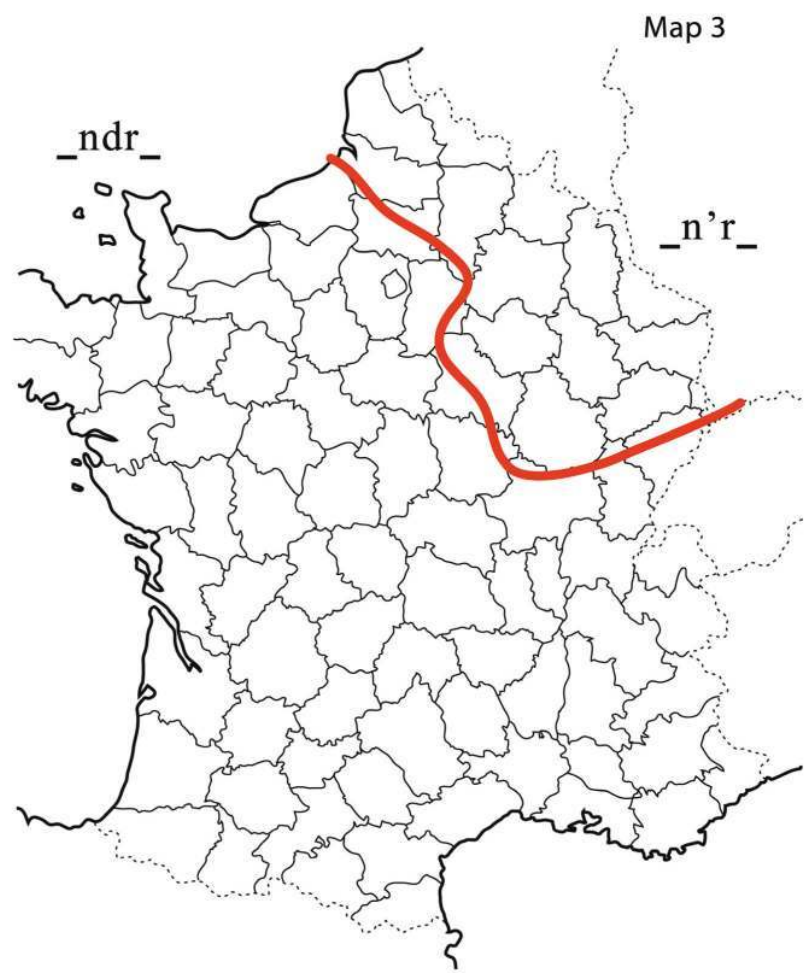

On trouvera d'autres exemples à : ensemble (ALF 464), gendre (ALF 634), moudre (ALF 879), poudre (ALF 1069), ressemble (ALF 1153), viendraient (ALF 1366). Voir Monjour (1989 : 137, $138,140,142)$ et Wüest $(1979 \mathrm{~b}: 319)$. Tandis que dans l'ouest et dans le centre, une consonne épenthétique s'est insérée dans les groupes [-nr-], [-ml-], et [-lr-], rien de tel ne se produit au nord et à l'est (voir Wüest 1979b : 315-320, Wüest 1985 : 245-6, Dees 1980 : 266, Pfister 1993 : 24-6, Fondet $1995: 193)$. Simoni-Aurembou (1999: 565) souligne « L'importance de cette frontière orientale » en se rapportant à des variables lexicales.

(D) Au long du côté méridional du cadre, nous pouvons placer les isoglosses occitanes comme la fermeture de [a] accentué en [e] : 


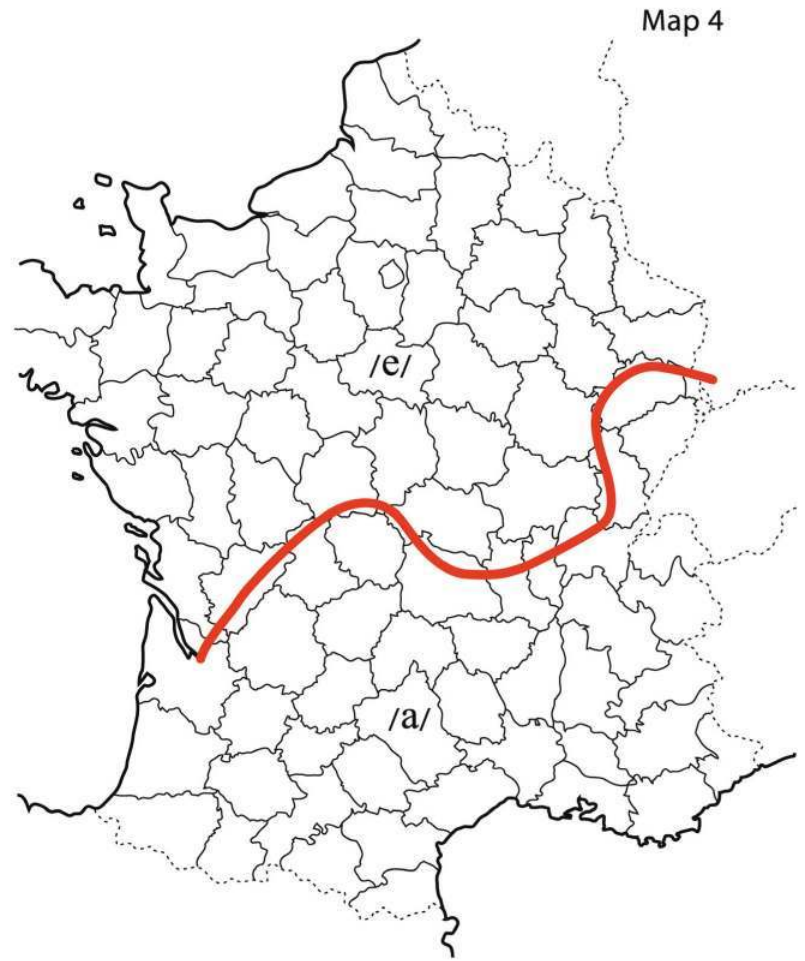

On trouvera d'autres exemples à : aile (ALF 18), échelle (ALF 436), noël (ALF 914), poteau ( ALF 1066), sel (ALF 1213). La fermeture de [a] accentué en [e] différencie les dialectes du nord gallo-roman de ceux du sud (voir Wüest 1979b : 170). On remarquera toutefois qu'à l'est de la langue d'oil, [a] dans cette position se diphtongue en [aj] (voir Pfister 1993 : 35-6), et qu'à l'ouest, [a] demeure devant [1].

Si nous superposons ces quatre isoglosses, nous créons un cadre autour de l'Ile-de France et de l'Orléanais qui correspond grosso modo à la zone IV de Bec (1971, t.2). 


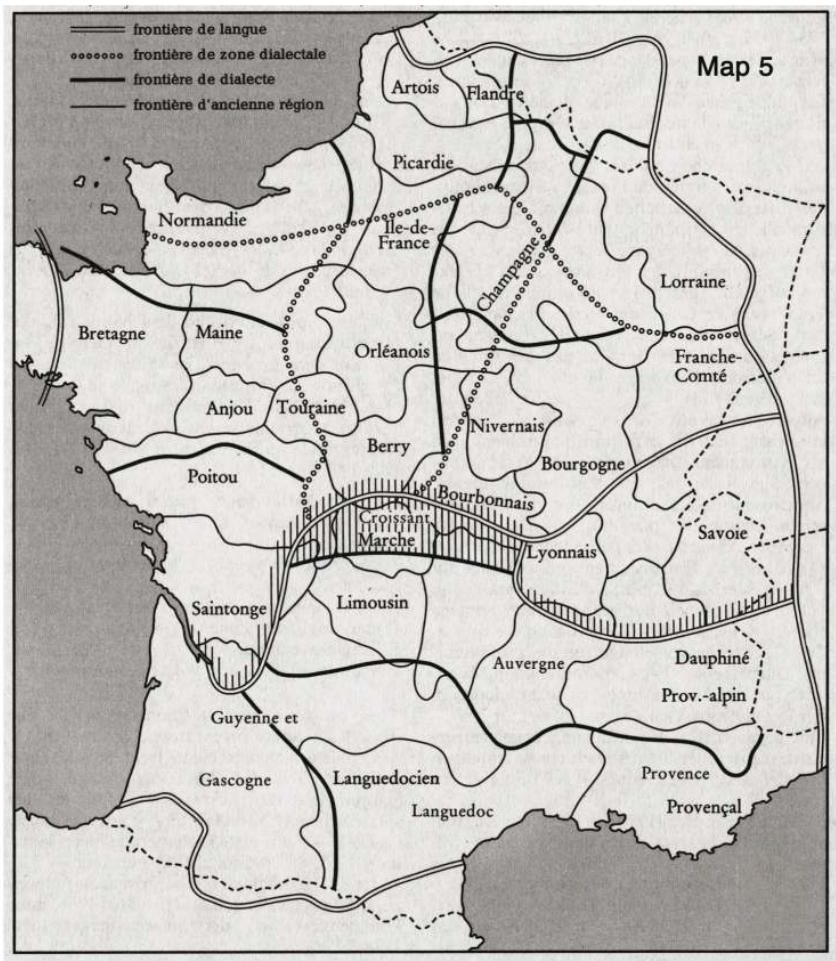

Il convient de souligner que ces quatre isoglosses ne sont pas à considérer comme des frontières dialectales fermes - à l'exception peut-être de l'isoglosse (D), on ne peut trouver un nombre significatif d'autres isoglosses qui coïncident avec celles illustrées ici. Cela n'est pas surprenant, et est même tout à fait prévisible dans la situation de continuum dialectal qui est celle de l'aire dialectale du nord gallo-roman. La partie nord se fond dans le picard, l'ouest dans le normand, l'est dans le champenois, etc. Il faut reconnaitre aussi que l'emplacement actuel des bords de notre cadre n'est probablement pas co-extensif avec celui des siècles passés. Il est plus que probable que le poids démographique de Paris a eu pour effet de pousser les isoglosses vers la périphérie. On constate tout de même d'une part que les parlers situés à l'intérieur du cadre partagent ces traits, d'autre part qu'ils sont aussi présents dans ce qui est devenu le français standard.

Malgré la présence de ces traits communs, l'espace dialectal à l'intérieur du cadre n'est pas homogène : on observe plusieurs exemples de ce qu'une forme externe pénètre le centre de la zone pour former une saillie dans la région parisienne, et on constate que ces traits appartiennent aussi au français standard. Dans ce qui suit, nous traiterons cinq de ces traits, dont quatre ont déjà été signalés dans l'étude de Fondet (1995).

\section{(1) [wa] [we]}




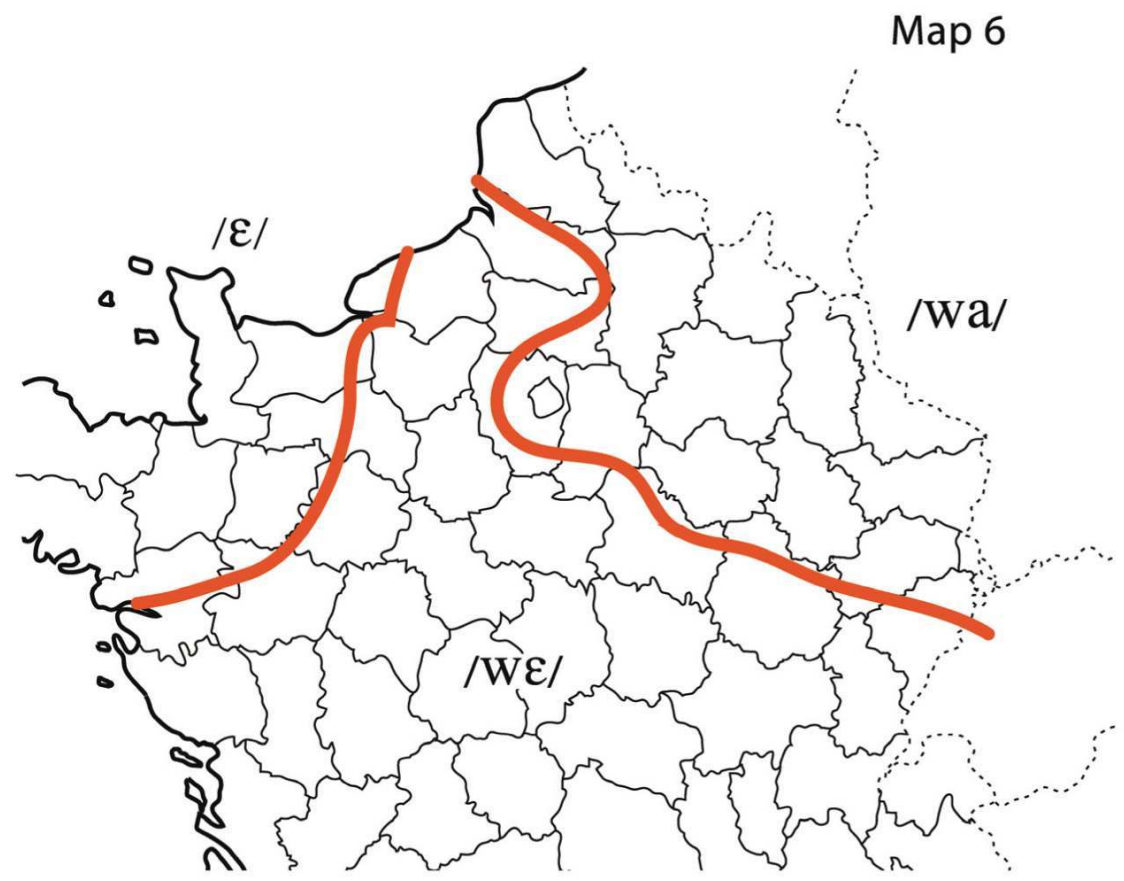

On trouvera d'autres exemples à : soir (ALF 1238), droit (ALF 428), endroit (ALF 460), froid ( ALF 612). Voir aussi Wüest (1979b: 203) et Monjour (1989: 98). Nous voyons ici une concentration de formes en [wa] à l'est de notre zone et une saillie vers l'ouest de la région parisienne. Il s'agit là d'une des variables phonétiques de la langue d'oïl les plus sujettes à la variation et au changement. Bien avant le XII ${ }^{\mathrm{e}}$ siècle, le [e/i] latin accentué s'était diphtongué en [ej] dans tout le nord gallo-roman, fusionnant avec la diphtongue provenant de [e] accentué + palatal. Tandis que dans l'ouest cette diphtongue est ensuite devenue $\rightarrow[\mathrm{e}]$ (dans certaines régions $\rightarrow$ [a]), dans l'est elle est devenue [oj] $\rightarrow$ [we] (voir Haudricourt $1948: 212$ ), et dans certains endroits [wa] (voir Wüest 1979b : 198-204, 379 et Pfister 1993 : 29-30).

(2) [jo] [o] 


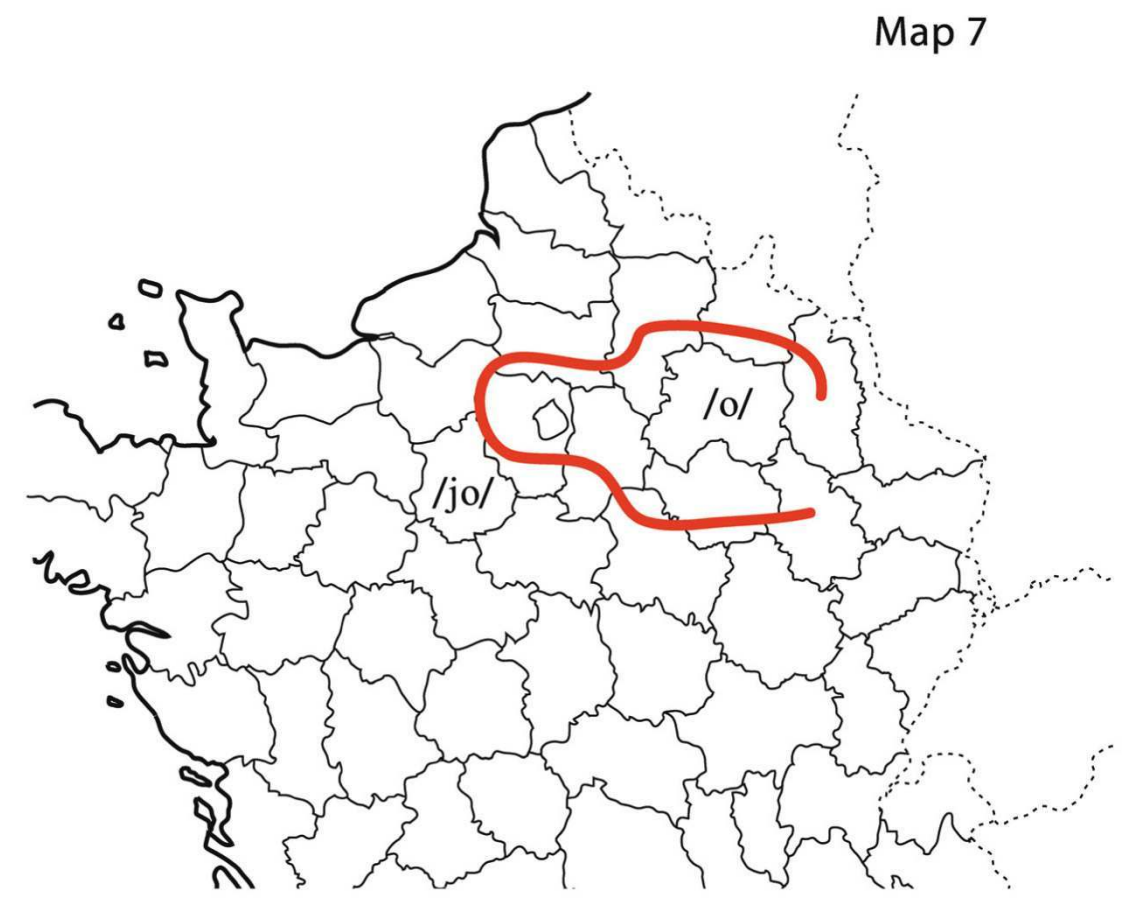

On trouvera d'autres exemples à : seau (ALF 1208), eau (ALF 432). Nous sommes ici en présence d'une forte concentration de formes en [o] dans l'est avec une saillie vers l'ouest de la région parisienne. En ancien français, la vocalisation de [1] devant [s] final dans le groupe -els (< latin -ellos) amena l'insertion d'un [a] épenthétique et la formation d'une triphtongue [eaw]. Les données de l'ALF indiquent que cette triphtongue a évolué différemment selon les dialectes. La plupart d'entre elles présentent la forme différenciée [jo], résultant d'une mutation consonantique du premier élément $[\mathrm{e}]>[\mathrm{j}]$, ainsi qu'un nivellement des deux éléments suivants $>$ [o]. Dans la région parisienne et dans une zone assez restreinte à l'est du pays, nous trouvons la simple monophtongue [o], qui se serait développée sans différentiation à partir de l'ancien français [eaw] $>[\mathrm{eo}]>[\mathrm{o}]$ (voir Pope 1935 : 200-201). Le rapport historique entre les deux variantes dans le parler parisien n'a pour l'instant pas été clairement élucidé. Dauzat (1927:40) considère que la variante non différenciée $[\mathrm{o}]$ < [eo] du français de Paris est la forme indigène, et que la variante [jo] est une forme rustique qui n'a jamais réussi à s'implanter vraiment dans la capitale. Fouché (1952: 336) voit [o] < [eo] comme une variante créée de toutes pièces par les classes supérieures pour se distinguer des prolétaires. Wüest (1985: 243) est du même avis, signalant l'emploi presque général de la graphie « iau » dans les textes parisiens du XIII ${ }^{e}$ siècle. Il conclut que « la différence entre eau et iau ne pouvait donc être dialectale; elle devait être sociolectale. » L'origine de la variante indifférenciée $([\mathrm{o}]<[\mathrm{eo}]<[$ eaw $])$ reste néanmoins incertaine. On peut y voir une innovation endogène dans le parler des classes supérieures, mais la concentration de formes monophtonguales dans une région située à l'est de Paris laisse ouverte la possibilité d'une origine exogène.

(3) -ent -ont/-ant (6e personne du présent de l'indicatif) 


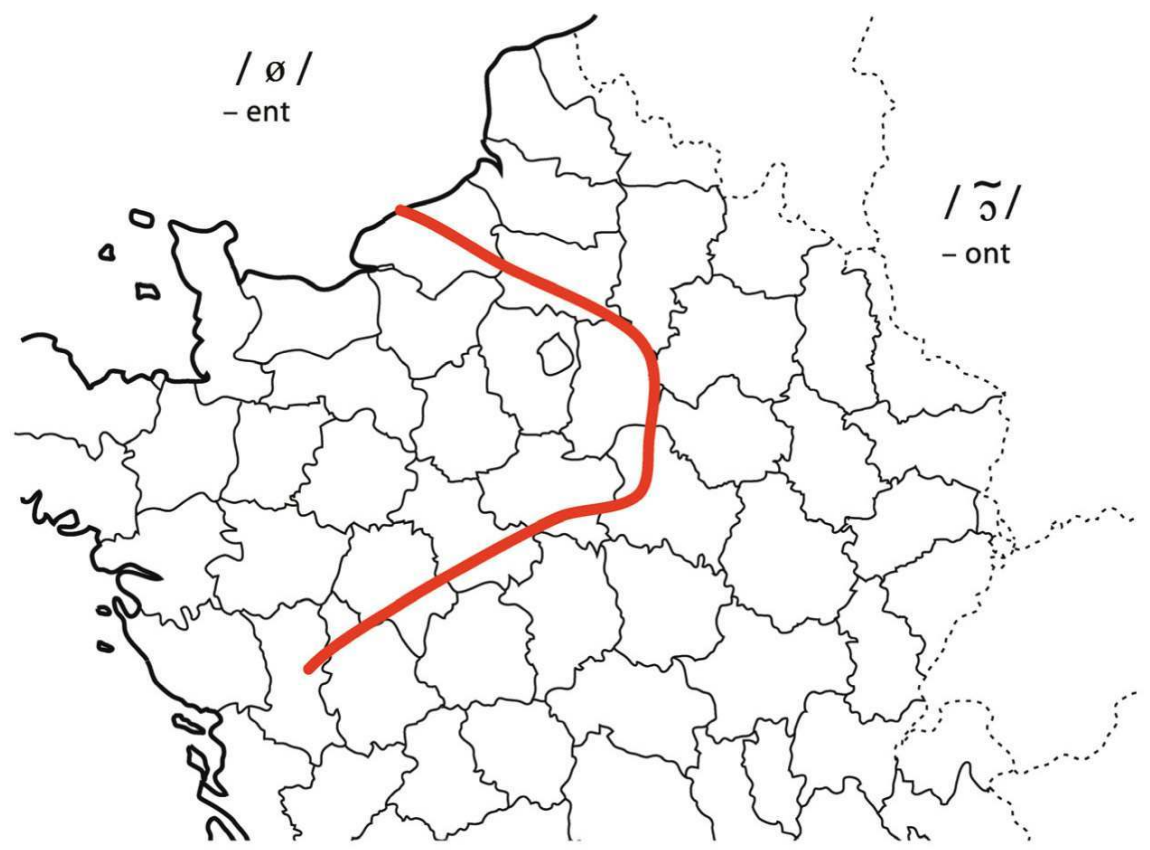

On trouvera d'autres exemples dans $A L F, 311,574,679$. Nous constatons ici une forte concentration de formes en -ent dans l'ouest et une saillie vers l'est dans la région parisienne. Les vernaculaires traditionnels $d u$ nord gallo-roman présentent deux traitements de la terminaison de la $6^{\mathrm{e}}$ personne : des variantes où l'accent se place sur la terminaison (les formes en -ont, -ant) et d'autres, localisées surtout dans l'ouest, où la terminaison est sans accent (les formes en -ent) (voir Wüest 1985 : 234-5). Fouché (1967 : 194-5) ne dit rien sur l'évolution de ces terminaisons en -ont/-ont. La terminaison -ent semble s'être diffusée assez rapidement dans la langue écrite. Pope (1952: 385) trouve néanmoins des exemples sporadiques -ont, -ant ainsi qu'une troisième variante -ient dans des manuscrits médiévaux rédigés hors des aires centrales et occidentales. Fondet (1980: 70-2) note la présence de -ont dans le parler de l'Ile-de-France au XVI ${ }^{\mathrm{e}}$ siècle : «au XVI siècle la finale dialectale -ont est bien attestée ; mais l'extension considérable de ce trait interdit d'y voir un phénomène récent ».

(4) -ions -iens (4e personne du subjonctif présent et de l'imparfait de l'indicatif) 


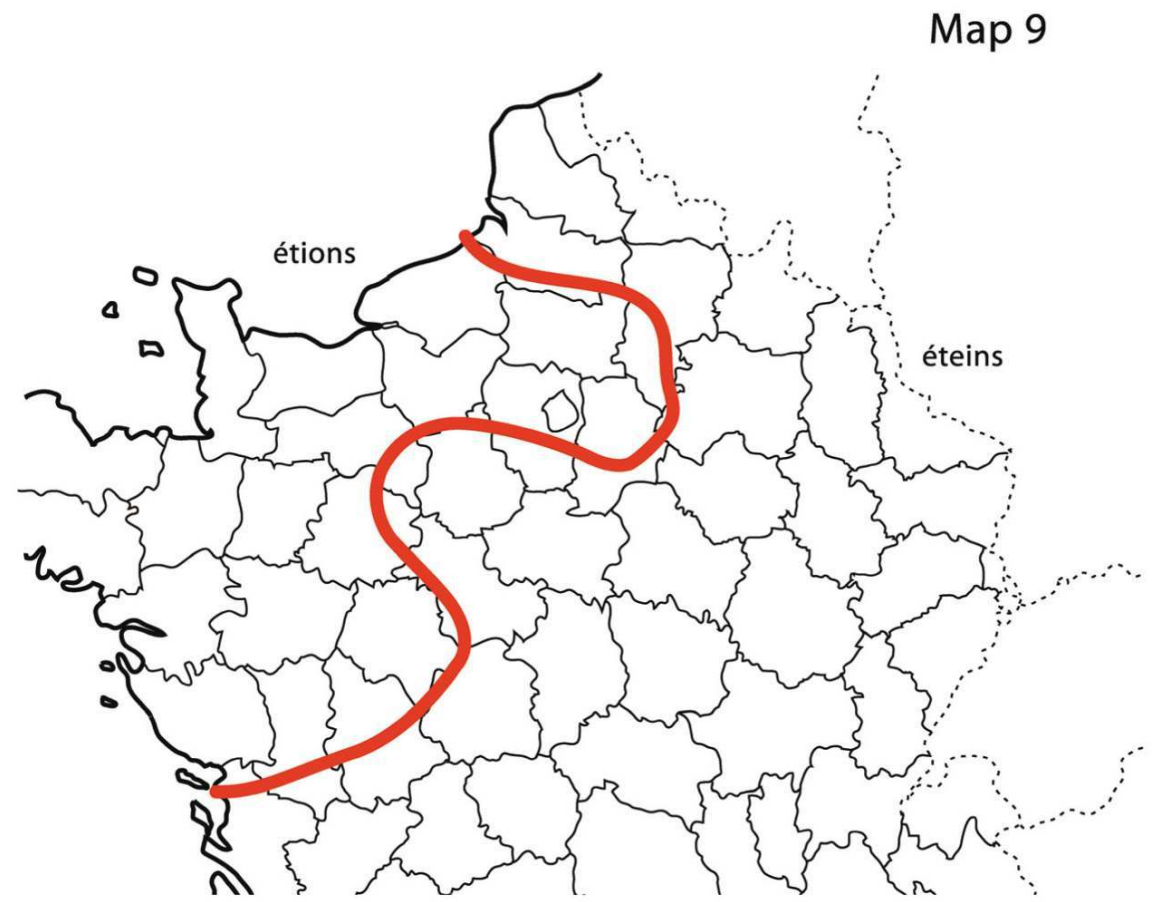

On trouvera d'autres exemples dans ALF 100, 515. Nous constatons ici une forte concentration de formes en -ions à l'ouest ainsi qu'une saillie vers l'est de la région parisienne. Fouché $(1967: 239,242-3)$ considère que la terminaison en -iens avait été générale sur toute la zone du nord gallo-roman dès "le français le plus ancien ». C'est dans la Chanson de Roland qu'il trouve les attestations les plus anciennes de terminaisons renfermant une voyelle postérieure arrondie (-ium). Pope (1935: 347) fait observer que «In the western region -iiens was replaced by -iuns (-ium etc.) in the twelfth century ... and in the thirteenth century these forms become general in the central region. » Voir aussi Fondet (1995 : 195).

(5) -aient $\sim$-aint (6e personne de l'imparfait et du conditionnel) 


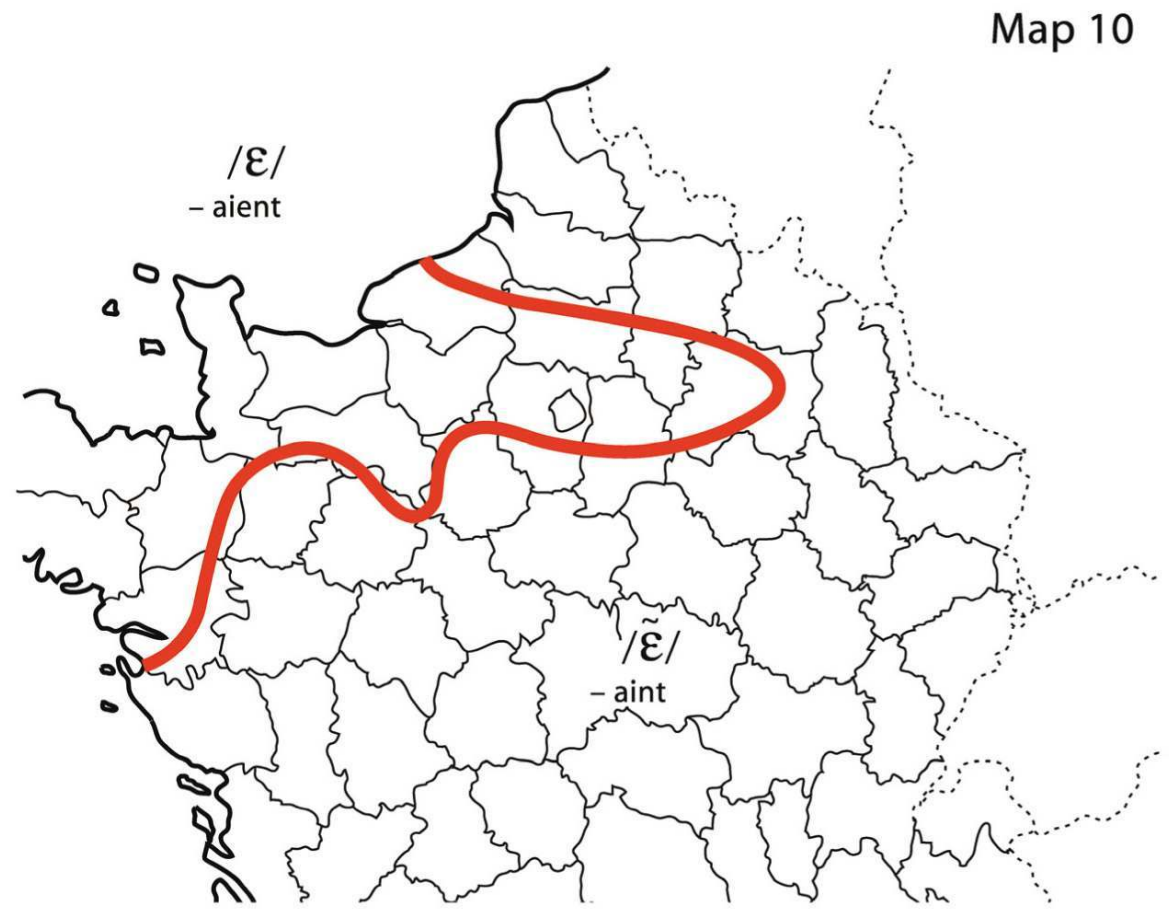

On trouvera d'autres exemples dans ALF 401, 513, 1366. Fondet (1995: 195) décrit « une vaste partie du domaine d'oill où « un imparfait de type -ebam, etc., [est] resté au stade / ei/, avec nasalisation à la troisième personne du pluriel. » Fouché (1967 : 241) attribue les formes en -aint spécifiquement à l'Orléanais et les considère comme "très anciennes, antérieures à la nasalisation de la diphtongue ei et refaites sur la $3^{\mathrm{e}}$ pers. sing. -eit) ». Pope (1935 : 496) attribue ces formes à la région de l'est. Wüest (1985: 234) est frappé par la « répartition assez bizarre de ces formes». En dépit du manque d'unanimité chez ces éminents chercheurs, la carte 10 offre une forte concentration de formes en -aient dans l'ouest et une saillie vers l'est de la région parisienne.

La présence de configurations en saillie dans la distribution de ces cinq isoglosses n'est pas sans importance pour l'histoire linguistique de la région. Elle suggère que dans ces cinq cas, une variante exogène a pris racine d'abord dans le parler parisien, d'où elle a été diffusée dans la campagne environnante. La diffusion de ces formes dans les vernaculaires ruraux autour de Paris ne s'est pas faite du jour au lendemain. Les atlas linguistiques par régions publiés à partir des années 1960 (l'ALIFO, voir Simoni-Aurembou (1973a), l'ALN, voir Brasseur (1980) et l'ALCB, voir Bourcelot (1966)), nous offrent souvent des formes reliques isolées à l'intérieur de cet espace qui remontent à une époque antérieure à la diffusion vers l'extérieur de ces façons de parler urbaines. C'est ce qui ressort d'ailleurs des documents rédigés entre le XVII ${ }^{\mathrm{e}}$ et le XIX ${ }^{\mathrm{e}}$ siècle, qui nous offrent des indices précieux concernant le parler de l'arrière pays immédiat de Paris avant l'effacement des vernaculaires ruraux traditionnels au cours du vingtième siècle. Les documents que nous avons consultés au cours de cette étude sont les suivants (classés par ordre chronologique inverse) : 
Passy (1891) : « Les villages de la vallée du ru de Gally, petit affluent de la Maudre, ont conservé jusqu'à ce jour un parler assez différent du parisien et du français d'école. » (Sainte-Jamme, Yvelines, à l'ouest de Saint-Germain-en-Laye)

Agnel (1855): « j'étais frappé de ces différences entre la prononciation de notre langue du XIX ${ }^{e}$ siècle, exactement parlée, et la prononciation du langage rustique. » ( « département de Seine-et-Oise »)

Lettres de Montmartre (1750) - Montmartre (Seine-Saint-Denis)

Sarcelades (1730-48) - Sarcelles (Val d'Oise)

Agréables Conférences (1649) - Saint-Ouen et Montmorency (Val d'Oise) voar. bourgeas seau ». » chapiaux. drappiau. voyaint fezien "saillie parisienne»?

\section{(1) - [wa] [we], e.g. poire, droit, froid}

Passy (1891: 16) : "Ordinairement on a wé ou wè : mwé ou mwè «moi », bwét « boîte », swéf « soif, frwésé « froisser » .... »

Agnel (1855 : 15-16) : «Dans la prononciation rustique la syllabe oi se change en oué. Par exemple, on dit la loué, la souéf, le pouél ...»

Lettres de Montmartre (1750) : comporte de nombreuses formes du type dret, dait,

Sarcelades (1730) : pas d'exemple.

Agréables Conférences (1649): comporte de nombreuses formes du type touay, foa,

\section{(2) - [jo] [o], e.g. manteau, seau, eau}

Passy (1891:16) : «Le groupe graphique -eau est ordinairement représenté par ó et non pas par yó comme on aurait pu l'attendre. Cependant on dit toujours é syó « un

Agnel (1855: 13) : «Dans le langage rustique, la syllabe finale eau se prononce iau. Tel est le principe général. Toutefois cette prononciation tombe de jour en jour en désuétude dans les environs de Paris et n'est conservée que dans quelques mots comme çiau, musiau, biau pour seau, museau, beau. Mais lorsque l'on atteint les arrondissements d'Etampes, de Pontoise et de Mantes ou les départements de l'Oise et de Seine-et-Marne, cette prononciation de la syllabe iau, dans les mots finissant en eau, reparaît en entier et s'emploie généralement. »

Lettres de Montmartre (1750): comporte de nombreuses formes du type biaux,

Sarcelades (1730): comporte de nombreuses formes du type troupiau, chapiau.

Agréables Conférences (1649): comporte de nombreuses formes du type gastiau,

\section{(3) - (-ent -ont) 6e personne de l'indicatif :}

Passy (1891) : aucun exemple

Agnel (1855) : aucun exemple

Lettres de Montmartre (1750) : comporte de nombreuses formes du type changiant,

Sarcelades (1730) : comporte de nombreuses formes du type appellont, chantont

Agréables Conférences (1649) : comporte de nombreuses formes du type bouton, scavan

(4) - (-ions -iens) 4e personne de l'imparfait de l'indicatif, etc. :

Passy (1891) : aucun exemple

Agnel (1855) : 54 j'avions ou javiens, 60 j'étions ou j'étiens

Lettres de Montmartre (1750) : aucun exemple

Sarcelades (1730) : aucun exemple

Agréables Conférences (1649): comporte de nombreuses formes du type j'alien, je

\section{(5) - (-aient -aint) 6e personne de l'imparfait de l'indicatif :}


Passy (1891: 10) ín kúpré pá sa (« ils ne couperaient pas ça »)

Agnel (1855: 54) ils avaient ou ils avient, 61 il étiont ou il étient

Lettres de Montmartre (1750) : comporte de nombreuses formes du type aviaint, etaint

Sarcelades (1730) : aucun exemple

Agréables Conférences (1649): comporte de nombreuses formes du type laissient,

attendien

45

âce au travail de Claire Fondet, rien de tout cela n'est entièrement nouveau, mais il est important de signaler que ces faits ne vont pas sans conséquences pour l'évolution du parler de Paris et des normes du français standard. Ces remarques nous révèlent de manière nette l'évolution plus rapide du parler urbain par rapport à celui de la campagne, et la longue persistance, dans les environs immédiats de Paris, de formes remontant à une époque antérieure à la diffusion du français standard à partir de la ville. Il est évident

- que la langue standard est fondée sur le parler de l'Ile de France (variables (A) - (D)).

- qu'elle comporte de nombreux éléments importés de l'extérieur (variables (1) - (5)).

- que ce mélange de formes a pris racine dans le parler de la grande ville avant d'être diffusé vers la périphérie.

Le français standard (qui équivaut ici au "français de Paris ») n'est pas un simple prolongement historique du dialecte parlé de l'Ile-de-France; elle en représente plutôt une forme modifiée, une variété mixte, une « koinè » (pour reprendre un terme utilisé par les créolistes).

Celui-ci peut ne pas convenir à certains lecteurs, car différents aspects de la "koinè » originale du grec ancien ont été pris comme traits définitoires par différents linguistes. Pour Trask (1997 : 122) il désigne

a more-or-less uniform spoken language which is used as the ordinary vehicle of communication over a sizeable area, usually by a number of people for whom it is not the mother tongue.

Dans un sens similaire, ce terme a été appliqué à une langue littéraire supra-régionale utilisée dans le nord de la France au cours du Moyen Age (voir Wacker 1916). Ces emplois du terme doublent respectivement le sens des expressions «lingua franca » et «norme littéraire ", enlevant ainsi à « koinè » une partie de sa valeur. Les créolistes utilisent ce terme d'une manière différente. Pour eux il désigne, comme la «lingua franca», une variété de langue utilisée pour la communication inter-dialectale or inter-linguale, mais une koinè possède en plus la propriété essentielle d'être un mélange de langues ou de dialectes en situation de contact, amenant le développement d'une variété nouvelle (voir Siegel 1985). Comme le dit Trudgill (1986:107-8), une koinè est

a historically mixed but synchronically stable dialect which contains elements from the different dialects that went into the mixture, as well as interdialect forms that were present in none,

les processus de base étant le mélange dialectal, le nivellement et la stabilisation. C'est en ce dernier sens que nous utilisons le terme.

Si le français standard (ici « français de Paris ») est à l'origine une sorte de koinè, au sens créoliste, comment expliquer son développement? Nous nous trouvons face à deux lignes d'approche. Il peut provenir d'une koinè écrite fabriquée de toutes pièces et imposée par la suite comme norme orale, ou bien il peut remonter à une koinè orale développée spontanément par les locuteurs et par la suite élaborée en langue standard. Nous appellerons ces deux approches respectivement: Hypothèse de la Koinè Ecrite et Hypothèse de la Koinè Orale. Le choix entre elles est délicat, car il dépend de l'interprétation des données écrites du Moyen Age et de la position que nous choisissons d'adopter envers l'un des problèmes les plus difficiles de la philologie médiévale, le 
rapport entre graphème et phonème dans les manuscrits d'ancien français (voir Wacker 1916, Robson 1955).

\section{I'hypothèse de la koinè écrite}

koinè élaborée à l'écrit à une date ancienne. Elle puise ses racines dans la «Skriptatheorie » énoncée par C.-T. Gossen dans les années 1960 pour rendre compte du rapport graphème-phonème dans le système d'écriture de l'ancien français (voir Gossen 1962, 1967).

Bien que la suppression de la variation soit infiniment plus facile à atteindre à l'écrit qu'à l'oral, le degré d'uniformité graphique visible dans les textes de l'ancien français est loin de ce que nous trouvons dans les textes modernes. Les textes vernaculaires de l'époque étaient rédigés par des gens fort éloignés de l'idée moderne d'uniformité de la langue: chaque texte renfermait habituellement des traits appartenant en propre à des régions différentes de celle dans laquelle il avait été écrit. C'est notamment le cas des textes littéraires, les poètes recourant souvent à des formes empruntées à d'autres dialectes aux seules fins de satisfaire les nécessités du mètre et de la rime, et les copistes ultérieurs modernisant, souvent de manière non systématique, la graphie de manuscrits pour se mettre à l'heure des modes du jour. D'ailleurs, les textes littéraires étaient destinés à circuler (voir Poerck 1963:2), si bien qu'ils présentaient une véritable superposition de traits linguistiques propres à d'autres régions que celle où ils avaient été composés. On notera pourtant que même les textes statiques que sont les chartes et les documents administratifs contiennent eux aussi un grand mélange de formes dialectales (voir Monfrin 1968). Comment expliquer cette variabilité? l'ancien français ont été vues d'un côté comme un système graphémique/phonémique, où les unités de base sont des lettres qui représentent des sons individuels d'une manière relativement directe, et de l'autre côté comme un système au fond logographique, où les unités de base sont des ensembles de lettres représentant des mots complets et n'ayant qu'un rapport assez ténu avec la prononciation. Il y a un siècle, les attitudes envers les graphies médiévales étaient dominées par une vision romantique du moyen âge, celle d'une culture naïve et spontanée : à mesure qu'émergeait un nouveau système d'écriture vernaculaire aux $\mathrm{X}^{\mathrm{e}}-\mathrm{XI}^{\mathrm{e}}$ siècles, les scribes auraient été attirés tout naturellement vers le système le plus transparent (le système graphémique/phonémique). Le système d'écriture reflétait les sons individuels de manière simple et directe. La variation au sein d'un même manuscrit était causée par les interférences avec un dialecte voisin, l'auteur ou scribe étant supposé avoir vécu près d'une frontière dialectale ou s'être déplacé entre aires dialectales. Avec la marche du temps, la civilisation médiévale aurait perdu un peu de sa fraîcheur, de sorte que vers la fin du Moyen Age, les graphies vernaculaires, comme l'art médiéval en général, se seraient vues soumises à des conventions sclérosées et seraient progressivement devenues plus logographiques.

53 Les spécialistes du $\mathrm{XX}^{\mathrm{e}}$ siècle ont remis sérieusement en question cette façon de voir. L'idée typiquement romantique d'une culture médiévale naturelle et spontanée fut remplacée par une conception selon laquelle elle aurait été entièrement gouvernée par des conventions implicites. Chez les linguistes, les méthodes néo-grammairiennes ont 
persisté pendant plusieurs décennies, mais vers le milieu du siècle, sans doute sous l'influence d'une conception saussurienne selon laquelle «langue et écriture sont deux systèmes de signes distincts» (Saussure 1971: 45), le système graphique de l'ancien français a commencé à être vu comme un système fermé qui fonctionnait selon ses lois internes propres purement visuelles, n'ayant qu'un rapport limité avec la langue parlée. Tout en laissant une petite place au modèle graphémique/phonémique dans les graphies des textes les plus anciens, on commençait à voir dans les graphies utilisées dans les textes rédigés à partir du XII ${ }^{e}$ siècle un système de plus en plus opaque et arbitraire, dicté non pas par une prononciation locale mais par des conventions implicites développées par un cercle relativement restreint de copistes travaillant dans un nombre limité de scriptoria. On en est venu à considérer le système graphique de l'ancien français comme étant beaucoup moins sujet à la variation qu'on l'avait pensé précédemment. Pour la zone de la langue d'oil au moins, tous les textes étaient censés se conformer à une norme écrite supra-régionale qui n'admettait qu'un nombre limité de régionalismes. Cette norme ne reposait sur aucun dialecte parlé, mais existait sur le seul plan visuel comme une "scripta». Le naïf positivisme du XIXe siècle fut remplacé par un scepticisme "postmoderne » à l'égard de la valeur référentielle des données écrites. Le linguiste se vit donc obligé d'abandonner le recours aux graphies dans la reconstruction de la langue parlée au Moyen Age, ce qui limitait considérablement la contribution qu'il pouvait offrir à l'une des questions centrales de la linguistique historique, le changement phonétique.

Cette approche, préfigurée par Wacker (1916), développée par Remacle (1948), fut systématisée par Gossen (1967) dans la "Skriptatheorie ", qui continue à être celle de nombreux chercheurs (voir Eloy 1997 : 53-8). On en est venu à croire qu'une langue standard écrite composite a émergé dans la langue d'oil indépendamment de la variabilité des parlers locaux à une époque antérieure au XII siècle. Delbouille (1970) a situé l'apparition de cette norme supra-régionale au IX siècle, avant la diversification des dialectes de la langue d'oil. Hilty (1973) l'a rattachée au parler de Paris au VIII e siècle. Cerquiglini (1993) suit le mouvement en soutenant qu'une koinè conventionnelle écrite fut élaborée dès le IX $\mathrm{X}^{\mathrm{e}}$ siècle par un groupe de clercs et d'auteurs littéraires "éclairés » sans référence à une base dialectale quelle qu'elle soit :

C'est grâce à l'existence d'une société cléricale, guidée par une "lumière de raison", animée par les litterati désirant illustrer un bel usage littéraire de l'idiome roman, que dès les premiers textes est fondé et pratiqué un "illustre françois". (Cerquiglini $1993: 120)$.

Suivant en cela Balibar (1985), il voit à l'œuvre un grand projet à long terme conçu à l'époque carolingienne, ayant pour objet l'unification linguistique et politique du pays :

Une langue française transcendant la diversité des parlures, inscrite dans le projet d'une forme commune échappant pour des raisons politiques ou esthétiques, à l'échange local et quotidien. (Cerquiglini 1993 : 124).

Ainsi, selon l'Hypothèse de la Koinè Ecrite, on aurait sélectionné dès le $\mathrm{IX}^{\mathrm{e}}$ siècle des variantes phonétiques et morphologiques dans plusieurs traditions d'écriture de la France du nord, pour en faire une langue écrite plus ou moins standardisée qui devait faciliter la diffusion de textes littéraires et même de documents officiels. Tout en admettant une certaine latitude dans son application, cette koinè écrite aurait été bien implantée comme norme déjà avant le XII ${ }^{e}$ siècle, tant dans l'administration royale que chez les auteurs littéraires. 

étendue à la langue parlée. Ayant débuté simplement comme norme pour l'écrit, la koinè devait être adoptée comme norme orale par la cour du roi, et ensuite diffusée chez les Parisiens, à commencer par ceux qui cherchaient à améliorer leur niveau social et culturel :

Ce fut, en France, la langue littéraire de la scripta, qui, sans être artificielle, s'élabora dans des conditions sociologiques différentes de celle des idiomes populaires. A partir du XIII siècle, le parler urbain, stratifié, certes, mais proche de celui de la classe aisée, n'a cessé de s'opposer au parler rural jusqu'à la Révolution. (Fondet 1995 : 201).

Cette idée surprend un peu dans l'article de Claire Fondet, car elle contredit en un certain sens le reste de son article. Néanmoins, dans cette vision des choses, qui semble être implicite chez beaucoup d'auteurs, la langue écrite des textes d'ancien français, au lieu de refléter la langue parlée de l'époque, était, au contraire, en train de la dicter, selon un processus familier à ceux qui avaient connu les techniques d'apprentissage du français en France sous la Troisième République et après. Pour certains, une fois passée dans la bouche du peuple ignorant de Paris, cette langue cultivée de la classe aisée fut pervertie et corrompue, donnant naissance au malheureux vernaculaire connu plus tard sous le nom de français populaire.

Cette vision des choses peut être résumée dans le schéma suivant :

60 koinè écrite

61 确

62 norme écrite

$63 \quad$ 政

64 norme orale

65 政

66 vernaculaire parisien

67 Une telle hypothèse cadre bien avec la séparation de l'écrit et de l'oral chère aux structuralistes, et convient parfaitement à ceux qui préfèrent voir la culture entre les mains d'une caste de clercs et de litterati. On sait que la graphisation joue un rôle indispensable dans la standardisation des langues (voir Joseph 1987 : 32-42). Mais est-ce que cette hypothèse est réellement soutenable dans le cas du français médiéval ? On verra maintenant qu'elle se heurte à des problèmes, à nos yeux, incontournables: la chronologie des données, et la diffusion des innovations linguistiques dans la population. Prenons ces questions une par une.

Comme nous l'avons vu, les traces de mélange de dialectes et de nivellement dialectal visibles dans les plus anciens textes français ont conduit à conclure que les conventions d'un système d'écriture étaient déjà en place avant le XII siècle. Que les textes littéraires, normalement faits pour circuler, aient comporté à cette époque reculée des formes dialectales extérieures à leur lieu d'origine et qu'ils aient nivelé les formes senties comme trop fortement marquées sur le plan dialectal, cela n'a rien de surprenant. Les actes d'accommodation linguistique sont, au contraire, tout à fait normaux dans toute rencontre linguistique. Mais cela ne permet pas d'affirmer que ces tentatives d'accommodation linguistique se produisaient de manière autre qu'individuelle et ad hoc. Du point de vue logique, un texte comme les Serments de Strasbourg ne peut à lui seul 
témoigner de l'existence d'une koinè stable. D'ailleurs, les preuves textuelles de l'existence d'une koinè écrite stable à cette époque reculée font entièrement défaut. Le nombre de manuscrits français remontant à avant 1100, même 1200, est bien trop réduit pour suffire à établir la stabilisation d'une koinè écrite à cette époque lointaine (voir Pfister 1993). Il est d'ailleurs difficile de trouver un cadre institutionnel, cour ou administration royale, qui aurait pu fixer une telle koinè. L'administration royale, qui ne s'installa à Paris qu'au XII siècle, commença à se servir du vernaculaire comme langue écrite seulement vers le milieu du XIII e siècle (voir Giry 1894: 464-472). Dans une communication personnelle, Serge Lusignan de l'Université de Montréal m'écrit: «le français du roi s'est construit au XIV ${ }^{e}$ siècle à la chancellerie et à la cour de Charles V, dans un rapport d'échange avec le latin.»

A l'époque moderne, l'expression "français standard» se rapporte à une variété de langue superposée aux variétés régionales - comportant une phonologie, une morphologie, une syntaxe et un lexique - qui se trouve réalisée à l'oral aussi bien qu'à l'écrit. En tant que langue pré-standardisée, l'ancien français s'écarte nettement de ce modèle, d'une part par le fait que la fixation de normes pour l'oral appartient à une époque beaucoup plus récente (XVI ${ }^{\mathrm{e}}$-XVII ${ }^{\mathrm{e}}$ siècles), d'autre part par le fait que la normalisation de l'écrit en était encore à ses débuts. Burnley (1989: 23-4) décrit la situation en moyen anglais dans des termes très similaires. En revanche, les études de Dees sur la variabilité dans les documents écrits en français médiéval montrent clairement que la standardisation du système d'écriture n'a commencé à être une réalité qu'au XV siècle. Ses travaux quantitatifs sur des centaines de documents du XIII ${ }^{e}$ siècle, datés et localisés avec précision, amènent le chercheur hollandais à conclure qu'il n'existait pas de norme supra-régionale pour la langue écrite avant le XIVe siècle :

La notion de koinè écrite, ainsi que la notion corollaire de scripta régionale, n'ont aucune adéquation observationnelle pour la période antérieure à 1300 (Dees 1985 : 113).

Le deuxième problème sur lequel bute la "thèse de la koinè écrite » est celui de la diffusion des innovations à travers la population, à commencer par celle de Paris. L'attribution à des copistes monastiques du $\mathrm{X}^{\mathrm{e}}$ siècle d'un projet à long terme pour l'unification linguistique de la nation est tellement anachronique qu'elle ne doit pas retenir notre attention. L'idée qu'une langue écrite à moitié standardisée ait pu influencer les habitudes phonétiques et morphologiques d'une part importante de la population parisienne au cours de la période médiévale n'est guère plus convaincante. Bien que les fonctions de l'écriture ne se limitent pas nécessairement à un simple enregistrement de la parole, et que l'écriture puisse constituer un code autonome susceptible d'influencer la parole des locuteurs (voir Haugen 1966 : 163), on peut douter qu'avant le XVI ${ }^{e}$ siècle, le prestige et la notoriété des formes écrites vernaculaires aient été suffisamment élevés pour permettre que cette influence exerce des effets, même au niveau des élites.

71 Il est plus qu'évident que la scripta parisienne médiévale a profondément marqué l'évolution ultérieure des normes écrites, mais cela ne permet pas de penser qu'à cette même époque elle ait pu exercer une influence analogue sur l'oral d'une communauté de locuteurs alors largement analphabète. Il se peut fort bien que, dès la seconde moitié du XII ${ }^{e}$ siècle, on ait cultivé dans l'entourage du roi une variété acrolectale du français servant à distinguer les cortois des secteurs non nobles de la population (voir Delbouille 1962, Muscatine 1981). On conçoit facilement l'influence possible sur les formes 
linguistiques pratiquées par les écrivains de l'époque: fait clairement attesté dans le passage si souvent cité de Conon de Béthune, qui s'excuse de la présence dans son œuvre de traces d'un dialecte non approuvé par le cercle royal (voir Lodge 1997 : 137). Mais cela rend d'autant moins vraisemblable une forte influence linguistique s'exerçant en sens inverse, c'est à dire que des membres de la cour aient pu être amenés à imiter des formes linguistiques proposées par des troupes de jongleurs.

Le fait reste, néanmoins, que le mélange spécifique de formes dialectales que nous trouvons dans les textes parisiens du XIII ${ }^{\mathrm{e}}$ siècle a été par la suite adopté dans la langue parlée de la population parisienne, comme nous l'avons vu plus haut (voir \$3). On peut nourrir l'illusion que tout au long du Moyen Age litterati et Parisiens ordinaires habitaient deux planètes différentes et que ce que nous trouvons dans les textes d'archives représente un phénomène entièrement acrolectal et largement visuel, que la présence de la koinè dans les textes dramatiques (les Miracles par exemple) ainsi que dans des textes visiblement oraux comme les Cris de Paris (voir Milliot 1995) reflète une conspiration élaborée pour produire des écrits éloignés systématiquement du mode de performance. Chacun sait que le rapport entre graphème et phonème dans les textes écrits du Moyen Age n'était pas direct, et qu'il est impossible, à partir des graphies, de reconstituer en détail la langue parlée parisienne du XIII e siècle, mais il est trop facile d'en conclure qu'il n'y a pas eu de rapport du tout. Il est plus raisonnable de supposer avec Dees que la variation des systèmes d'écriture du XIII ${ }^{\mathrm{e}}$ siècle reflétait grosso modo la variation de la langue parlée de l'époque.

\section{I'hypothèse de la koinè orale}

73 L'hypothèse de la koinè orale suit l'opinion de Jacques Chaurand, entre autres, lorsqu'il affirme que les normes de la langue standard ne peuvent descendre directement du dialecte francien tel que le concevaient G. Paris, F. Brunot et alii. Elle s'écarte de ce qui semble devenir une orthodoxie actuelle en réfutant que la variété mixte qui se trouve à l'origine du français standard soit une langue conventionnelle écrite sortie tout armée de l'esprit d'un groupe d'auteurs littéraires et de planificateurs de langue avant l'heure. Elle soutient au contraire que cette variété s'est cristallisée spontanément au cours d'innombrables interactions quotidiennes entre des locuteurs réels. Les sources de la standardisation en français seraient à chercher principalement non dans une variété composite écrite, élaborée à date ancienne par une coterie de scribes, mais dans un dialecte mixte développé tout naturellement dans la langue parlée. La question est alors de savoir comment, quand et où un tel processus de koinéisation a pu se produire.

Les historiens du français font souvent allusion à un ancien français commun, dialecte partagé par tous les locuteurs de l'ancien français qui serait en quelque sorte le plus petit dénominateur commun de tous les dialectes de l'ancien français. En effet, rien n'interdit de supposer l'existence d'une telle langue (au sens saussurien du terme) à un niveau très abstrait. Le continuum dialectal du nord gallo-roman englobait un ensemble de variétés relativement proches entre lesquelles existait sans doute un haut degré d'intercompréhension. Cependant, lorsque nous poussons plus loin nos réflexions sur l' ancien français commun et sur l'espace sur lequel il a pu avoir cours, la notion devient moins facile à cerner qu'au premier abord. Est-ce qu'elle comprend les dialectes de la Gallo-Romania méridionale aussi bien que ceux du nord ? Pour des raisons descriptives et taxonomiques, le continuum dialectal gallo-roman est divisé traditionnellement entre 
langue d'oc et langue d'oil, mais il s'agit là, comme toujours avec les frontières dialectales, d'une fiction méthodologique (voir Chambers et Trudgill $1980: 103-123$ ). La dialectologie traditionnelle elle-même reconnaît l'existence de «zones de transition » entre la langue d'oc et la langue d'oil dans l'aire franco-provençale, dans le Croissant et en Poitou (voir Wüest 1979a). On peut penser que le classement moderne des langues romanes de la France médiévale, secondé bien souvent par des préoccupations identitaires, exagère la difficulté qu'éprouvaient les occitanophones à franchir la "frontière " linguistique avec la langue du nord, et inversement pour les Français septentrionaux. D'ailleurs, si nous choisissons d'exclure de l'ancien français commun les dialectes de la langue d'oc, pourquoi s'arrêter là ?On peut soutenir que les dialectes d'oil de l'Anjou avaient plus d'éléments en commun avec les dialectes d'oc du Poitou qu'avec les dialectes d'oil de la Picardie.Même à l'intérieur des dialectes affectés au gallo-roman du nord, quel pourcentage de l'ancien français commun était en effet partagé par les locuteurs, par exemple, du picard et de l'angevin?

75 Tout en souscrivant à l'idée abstraite d'une langue commune nord gallo-romane (voire gallo-romane tout court), lorsque nous considérons ses différentes réalisations au niveau de la parole, il est évident que nous avons affaire à un phénomène linguistique très éloigné de la notion de langue qui s'est développée dans l'ère post-standardisation, comportant des limites spatiales assez nettes et un niveau d'homogénéité interne assez élevé. Les recherches de Dees et de ses collaborateurs ont attiré l'attention à juste titre sur la variabilité inhérente de l'ancien français et ont démontré que cette variabilité est à analyser non en termes de déviations d'une norme centrale, mais de différences quantitatives dans la distribution des variables linguistiques clef. Durant les siècles qui suivirent la fragmentation sociale et économique de la Gaule romaine, il est plus que probable que les locuteurs de dialectes différents continuaient à se comprendre non en ayant recours à quelque langue commune pan-dialectale (ce qui impliquerait une situation de diglossie), mais à partir d'actes individuels d'accommodation, appliquant de manière ad hoc des séries de formules automatiques de conversion (voir Weinreich 1953).

Les notions de nivellement dialectal et de mélange de dialectes peuvent rebuter les partisans de "dialectes purs », qui y voient autant de cas d'« hybridation », mais c'est un truisme que d'affirmer que l'accommodation entre locuteurs de dialectes différents est une partie tout à fait normale de la vie quotidienne du langage. Quand les locuteurs provenant de secteurs non adjacents d'un continuum dialectal se trouvent en contact, il se forme des variétés mixtes plus ou moins temporaires. Il s'agit parfois de locuteurs (ou de textes) qui circulent dans différentes parties du pays, nivelant les traits les plus saillants de leur parler d'origine pour faciliter la communication. Il s'agit parfois de migrations de locuteurs de dialectes différents qui convergent sur un lieu central amenant en contact des formes de provenance différente et éliminant au cours de leurs interactions quotidiennes les formes régionales les plus marquées. Les locuteurs du galloroman se sont de tous temps déplacés sur leur territoire, accommodant à chaque rencontre leur parler dialectal à celui de leurs interlocuteurs. Toutefois, ces innombrables actes d'accommodation ne peuvent faire naître une koinè stable que lorsque certaines conditions sont remplies : les nouvelles koinès se forment en général après une période relativement longue d'interactions régulières et intensives, au cours de laquelle les actes individuels d'accommodation en viennent à s'orienter plus ou moins dans le même sens. Pour cela il faut normalement une focalisation significative des interactions sur quelque lieu central. 
77 Les sites privilégiés de telles densités d'interaction sont, on le conçoit facilement, les villes. Dans les siècles qui suivirent la chute de l'Empire romain, le réseau urbain hérité $\mathrm{du}$ monde antique s'était tellement fragmenté qu'il n'offrait plus les conditions nécessaires pour une focalisation dialectale quelque peu développée, au contraire. C'est seulement $\mathrm{au} \mathrm{X \textrm {I } ^ { \mathrm { e } }}$ siècle que les choses commencèrent à changer, avec un développement spectaculaire de l'urbanisation à travers toute l'Europe de l'ouest. Si le développement urbain a pris source en Italie du nord et en Flandre, et si la Picardie a vu une urbanisation tout à fait remarquable, au cours du XII ${ }^{\mathrm{e}}$ siècle une ville en particulier s'est distinguée sur le plan démographique de toutes les autres - Paris. Pour diverses raisons (voir Rauhut 1963), Paris à cette époque est partie de presque rien pour devenir une géante urbaine, éclipsant toutes les autres agglomérations du nord-ouest de l'Europe avec une population estimée à 100.000 en 1200, doublée un siècle plus tard.

Les plus grandes villes d'Europe, 1000-1900 (population en milliers)

\begin{tabular}{|c|c|c|c|c|c|c|c|}
\hline \multicolumn{2}{|c|}{1000} & \multicolumn{2}{|c|}{1400} & \multicolumn{3}{|c|}{1700} & 1900 \\
\hline Ville & $\begin{array}{l}\text { Popu- } \\
\text { lation }\end{array}$ & Ville & $\begin{array}{l}\text { Popu- } \\
\text { lation }\end{array}$ & Ville & $\begin{array}{l}\text { Popu- } \\
\text { lation }\end{array}$ & Ville & $\begin{array}{l}\text { Popu- } \\
\text { lation }\end{array}$ \\
\hline Constantinop & 450 & Paris & 275 & Constantinopl & 700 & Londres & 6,480 \\
\hline le & 450 & Milan & 125 & $\mathrm{e}$ & 550 & Paris & 3,330 \\
\hline Cordoue & 90 & Bruges & 125 & Londres & 530 & Berlin & 2,424 \\
\hline Séville & 75 & Venise & 110 & Paris & 207 & Vienne & 1,662 \\
\hline Palerme & 45 & Grenade & 100 & Naples & 188 & St.Petersbourg & 1,439 \\
\hline Kiev & 45 & Gênes & 100 & Lisbone & 172 & Manchester & 1,255 \\
\hline Venise & 40 & Prague & 95 & Amsterdam & 149 & Birmingham & 1,248 \\
\hline Regensbourg & 40 & Caffa & 85 & Rome & 144 & Moscou & 1,120 \\
\hline Thessaloniqu & 35 & Séville & 70 & Venise & 130 & Glasgow & 1,072 \\
\hline $\begin{array}{l}\text { e } \\
\text { Amalfi } \\
\text { Rome }\end{array}$ & 35 & Gand & 70 & $\begin{array}{l}\text { Moscou } \\
\text { Milan }\end{array}$ & 124 & Liverpool & 940 \\
\hline
\end{tabular}

(tiré de Hohenberg \& Lees 1985 : 11)

Il semble difficile, pour les historiens de la langue, de ne pas prendre en considération une évolution démographique de cette ampleur. Tout comme le Paris du XII ${ }^{e}$ siècle a restructuré la géographie et l'agriculture de la France du nord de manière radicale, il a eu un impact analogue sur la situation dialectale de la région. Il s'est produit à cette époque sur la place de Paris une augmentation exceptionnelle du taux d'interactions socioéconomiques et socio-linguistiques, entre Parisiens de souche et locuteurs provenant aussi bien de l'arrière-pays immédiat de la ville (connu depuis un siècle comme la zone francienne), que de régions plus éloignées (Normandie, Picardie, Champagne et au-delà). L'intensité et la régularité de ces interactions n'ont pu aboutir qu'à une nouvelle focalisation dialectale et à la stabilisation graduelle d'une variété urbaine qui finira par élever le parler de la ville au-dessus du continuum dialectal persistant dans les campagnes.

79 Si nous cherchons une variété koinéisée qui aurait servi de source première à la standardisation du français, on ne peut faire mieux que de la rattacher à la koinè orale amorcée vraisemblablement dans la métropole parisienne au XII ${ }^{e}$ siècle. Penny (2000 : 43-4) constate un développement analogue à Madrid lorsque cette ville devint capitale de l'Espagne au XVI siècle. Une telle hypothèse ne bute ni contre le problème de la chronologie des données écrites - Pfister (1973) a noté il y a déjà longtemps que c'est seulement au XII ${ }^{\mathrm{e}}$ siècle que le dialecte de Paris a commencé à acquérir un certain prestige - ni contre celui de la diffusion des innovations linguistiques à travers la 
population urbaine - si la koinéisation s'est produite dans le parler de la ville, cela explique du même coup la présence de traits non indigènes dans le français de Paris.

Ceci nous pousse à envisager le processus de la standardisation en français selon le schéma suivant :

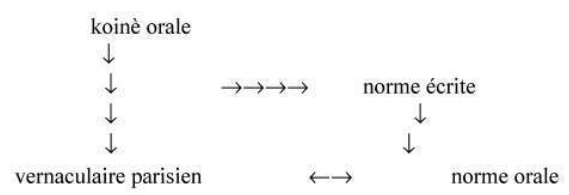

81 Dans ce modèle, la standardisation suit en France un parcours nettement plus complexe et plus intéressant que dans « l'Hypothèse de la Koinè Ecrite ». Il n'y a jamais eu au Moyen Age de projet directeur pour l'avenir de «la langue». La coalescence des normes de la langue standard s'est produite de manière empirique, presque aléatoire durant plusieurs siècles de concurrence entre variantes dans le parler quotidien de la grande ville. Il semblerait, par exemple, que des variantes exogènes morphologiques ont été absorbées bien avant certaines variantes phonologiques (telles la variante [eo] [jo]) (voir à ce propos Lass 1997 : 157). Koinéisation n'égale pas homogénéité. C'est seulement à l'époque de la Renaissance que l'imprimerie, alliée à une alphabétisation plus répandue, est parvenue à fixer les normes écrites de façon durable. Comme toujours, la standardisation de la langue écrite a précédé celle de la langue parlée, dont les normes ne furent fixées qu'au cours des XVII ${ }^{\mathrm{e}}$-XVIII ${ }^{\mathrm{e}}$ siècles, époque où elles étaient enfin susceptibles d'être influencées par la langue écrite.

Pour ce qui est du vernaculaire parisien moderne, il est légitime de penser qu'il y a eu continuité depuis la koinè orale du Moyen Age. Nous pouvons supposer qu'à l'origine « le français de Paris » et la langue standard embryonnaire étaient identiques, et qu'ils se sont séparés par la suite. C'est en tout cas l'avis d'Albert Dauzat (1927: 38). Au début de la période moderne, une nouvelle stratification sociale de la ville a eu comme contrecoup de placer la variété $\mathrm{H}$ sur un plan tout à fait différent de celui des variétés $\mathrm{B}$, et plus ou moins hors de portée des "habitants ignorants de la ville bornés à son seul horizon " (Cohen 1987: 185). A cette époque-là, les variantes alors en circulation furent «redistribuées» en tant que variantes diastratiques et diaphasiques - de manière inconsciente chez la masse des locuteurs, explicite chez les grammairiens et lexicographes. Ce processus de redistribution de variantes est bien attesté dans toutes les situations de contact entre dialectes et de koinéisation (voir Trudgill 1986:126). Au lieu de considérer le vernaculaire parisien comme une forme corrompue de la variété standard, comme le veut la tradition prescriptiviste, on ferait mieux de voir dans la variété standard un sociolecte créé (en partie au moins) pour distinguer le parler des élites de ceux du reste de la population urbaine.

\section{Conclusion}

83 Une tradition philologique plongeant ses racines dans le Romantisme voit dans la France médiévale une culture pré-industrielle et avant tout rurale, et tend à réduire au strict minimum le rôle linguistique des villes, en particulier celui de Paris. La cartographie traditionnelle des dialectes de l'ancien français ne fait aucune place au développement d'un dialecte urbain dans une métropole, qui, pourtant, dépassait de loin toutes les autres villes d'Europe sur le plan démographique, et cela dès le XIII ${ }^{e}$ siècle. J'ai soutenu dans cet 
article que l'urbanisation exceptionnelle de Paris durant le $\mathrm{XII}^{\mathrm{e}}$ et le $\mathrm{XIII}^{\mathrm{e}}$ siècles ne peut être négligée par les historiens de la langue : elle a créé un creuset linguistique immense et durable, un foyer tout indiqué pour les changements linguistiques rapides qui séparèrent progressivement le français de Paris des parlers dits « franciens » de l'arrièrepays rural. En cela Paris n'a fait que suivre au XII ${ }^{e}$ siècle des processus sociolinguistiques bien attestés dans de nombreuses grandes villes qui ont émergé dans le monde moderne (voir Manessy 1994).

Il nous semble que cette koinè parisienne orale constitue une base nettement plus solide pour la standardisation ultérieure du français que quelque koinè écrite, ancienne, mal documentée et, à l'époque médiévale en question, difficilement transmissible à la langue parlée. Avec la méthodologie variationniste proposée par Dees et ses collaborateurs, nous pouvons nous faire une idée approximative des éléments entrant dans la composition de cette koinè en étudiant la variabilité des documents (surtout administratifs) rédigés à Paris au XIII et au XIV ${ }^{e}$ siècles. Koinéisation et standardisation ne sont cependant pas la même chose. Il convient de concevoir le processus de standardisation en français comme complexe, tortueux et, surtout, prolongé. On pourrait même dire qu'il s'agit d'un processus continu qui suppose le maintien d'une tension permanente entre les facteurs qui favorisent l'unification de la langue et ceux qui au contraire y font obstacle. Une koinéisation originale a pu se produire dans la bouche de milliers de locuteurs parisiens, pour la plupart illettrés, mais la standardisation de la langue, qui va avec la graphisation, a dû encore attendre quelques siècles. Ce n'est qu'au début de l'époque moderne, lorsque les taux d'alphabétisation sont bien plus élevés, que les conditions sont réunies pour une réelle fixation des normes.

Il faut enfin se garder de considérer la standardisation du français comme un cas typique. En France, comme un peu plus tard en Angleterre, la longue domination démographique et économique d'une très grande ville - n'en déplaise à l'esprit girondin - a infléchi l'histoire de la standardisation en un sens nettement différent de celui qu'elle a pris ailleurs, en Allemagne et en Italie par exemple. Dans ces autres pays la focalisation socioéconomique et politique autour d'une ville capitale ne s'est jamais produite. Là effectivement le processus de standardisation, amorcé dès la Renaissance, a été construit, initialement au moins, sur la base d'une langue écrite supra-régionale. Mais la participation de la population à la culture écrite n'était plus alors ce qu'elle avait été à Paris trois siècles auparavant.

\section{BIBLIOGRAPHIE}

AGNEL, E. (1855) Observations sur la prononciation et le langage rustiques des environs de Paris, Paris : Schlesinger-Dumoulin.

Agréables Conférences de deux paysans de Saint-Ouen et de Montmorency sur les affaires du temps (1649-1651), Ed. F. Deloffre, 1999 (nouvelle édition augmentée d'une bibliographie complémentaire 1961-1998), Paris : Les Belles Lettres. 
BACON, R. (1897) «The Opus Majus » of Roger Bacon. J.S. Brewer (ed.), Oxford : Oxford University Press.

BADER, H. (1969) Von die "Francia" zur "Ile-de-France", Zurich : Wintertur.

BALIBAR, R. (1985) L'Institution du français, Paris : PUF.

BEC, P. (1971) Manuel Pratique de philologie romane, t.2, Paris : Picard.

BERGOUNIOUX, G. (1989) « Le francien (1815-1914) : la linguistique au service de la patrie », Mots/ Les langages du politique $19: 23-40$.

BOURCELOT, (1966) Atlas linguistique de la Champagne et de la Brie (ALCB), Paris : CNRS

BRUNOT, F. (1905) Histoire de la langue française, t.1, Paris : A. Colin.

BRASSEUR, P. (1980) Atlas linguistique de la Normandie (ALN), Paris : CNRS.

BURNLEY, D. (1989) The Language of Chaucer, Londres : Macmillan.

CERQUIGLINI, B. (1993) La Naissance du français, Paris : PUF (Que Sais-Je ?).

CHAMBERS, J. et TRUDGILL, P. (1980) Dialectology, Cambridge : Cambridge University Press.

CHAURAND, J. (1983) « Pour l'histoire du mot francien » In : Mélanges de dialectologie d'oil à la mémoire de R. Loriot, Dijon : ABDO, 91-99.

CHAURAND, J. (dir.) (1999) Nouvelle histoire de la langue française, Paris : Seuil.

CHAUVEAU, J.-P. (1989) Evolutions phonétiques en gallo, Paris : CNRS.

COHEN, M. (1987) Histoire d'une langue : le français, Paris : Les Editions sociales.

DAUZAT, A. (1922) La Géographie linguistique, Paris : Flammarion.

DAUZAT, A. (1927) Les Patois, Paris : Delagrave.

DEES, A. (1980) Atlas des formes et constructions des chartes françaises du $13^{e}$ siècle (ZRP, Beiheft 178), Tübingen : Niemeyer.

DEES, A. (1985) « Dialectes et scriptae à l'époque de l'ancien français », Revue de linguistique romane 49 : 87-117.

DEES, A. (1988) « Propositions for the study of Old French and its dialects », in J. Fisiak (ed.), Historical Dialectology (Trends in Linguistics, Studies and Monographs 17), 139-148.

DEES, A. (1989) « La reconstruction de l'ancien français parlé », in M.E.H. Schouten and P.T. van Reenen (dirs), New Methods in Dialectology, Dordrecht : Foris, 125-133.

DELBOUILLE, M. (1962) «La notion de "bon usage" en ancien français ", Cahiers de l'Association Internationale des Etudes Françaises $14: 10-24$.

DELBOUILLE, M. (1970) « Comment naquit la langue française? », in Mélanges offerts à M. Georges Straka. Lyon-Strasbourg : Société de linguistique romane, t. 1, 187-199.

ELOY, J.-M. (1997) La Constitution du Picard : une approche de la notion de langue, Louvain : Peeters.

FONDET, C. (1980) La Dialectologie de l'Essonne, Paris : Champion.

FONDET, C. (1995) « Contribution à la question des origines du français : quelques aperçus à partir de la dialectologie de l'Essonne », in M. Tamine (dir.), Ces Mots qui sont nos mots. Mélanges d'histoire de la langue française, de dialectologie et d'onomastique offerts au professeur Jacques Chaurand, Charleville-Mézières : Institut Charles Bruneau, 189-206.

FOUCHÉ, P. (1952) Phonétique historique du français, Paris : Klincksieck. 
FOUCHÉ, P. (1967) Le Verbe français, Paris : Klincksieck.

FOURQUIN, G. (1956) «La population de la région parisienne aux environs de 1328 », Le Moyen Age $62: 63-92$.

FOURQUIN, G. (1964) Les Campagnes de la région parisienne à la fin du Moyen Age, Paris : PUF.

FRANÇOIS, A. (1959) Histoire de la langue française cultivée, Genève : A. Jullien.

GAUTHIER, P. (1995) « Traits linguistiques communs », in Gauthier, P. and Lavoie, T. (dirs), 1995, 27-67.

GAUTHIER, P. and Lavoie, T. (1995) Français de France et français du Canada, Lyon : Université Lyon III.

GILLIÉRON, J. and EDMONT, E. (1901-11) Atlas linguistique de la France, (ALF). Paris : Champion.

GIRY, A. (1894) Manuel de Diplomatique, Paris : Hachette.

GOSSEN, C.-T. (1962) «Langues écrites du domaine d'oil », Revue de linguistique romane 26 : 271-308.

GOSSEN, C.-T. (1967) Französische Skriptastudien, Vienna : Österreichische Akademie der Wissenschaften.

HAUDRICOURT, J. (1948) « Problèmes de phonologie diachronique » (français EI > OI). Lingua 1 : 209-18.

HAUGEN, E. (1966) Dialect, language, nation. Repr. In J.B. Pride and J. Holmes, Sociolinguistics. Harmondsworth : Penguin, 1972.

HILTY, G. (1973) Les origines de la langue littéraire française, Vox Romanica 32 : 254-71.

HOHENBERG, P. M. and Lees, L. H. (1985) The Making of Urban Europe 1000-1950, Cambridge Mass. : Harvard University Press.

JOSEPH, J. E. (1987) Eloquence and Power. The Rise of Language Standards and Standard Languages, Londres : Frances Pinter.

LABOV, W. (1994) Principles of Linguistic Change, Vol.1 (Internal Factors). Oxford : Blackwell.

LASS, R. (1997) Historical Linguistics and Language Change, Cambridge : Cambridge University Press.

Lettres de Montmartre (1750) « à Londres », J. Georgin (= A.-U. Coustelier)

LODGE, R. A. (1997) Le Français. Histoire d'un dialecte devenu langue. Paris : Fayard.

MANESSY, G. (1994) « Modes de structuration des parlers urbains », in E. Elhousseine and N. Thiam (dirs), Des Langues et des villes, Paris : Didier Erudition, 7-27.

MATZKE, E. (1880-1881) « Der Dialekt von Ile-de-France im XIII und XIV Jahrhundert », Archiv für das Studium der neuren Sprachen und Literaturen $64: 385-412,65: 57-96$.

MICHAËLSSON, K. (1959) «Quelques variantes notées dans la prononciation parisienne au temps de Philippe le Bel ». VIII Congresso Internazionale di Studi Romanzi, Florence, II.2, 287-297.

MILLIOT, V. (1995) Les Cris de Paris ou le peuple travesti. Les représentations des petits métiers parisiens (XVI ${ }^{e}-X V I I I^{e}$ siècles). Paris : Publications de la Sorbonne.

MONFRIN, J. (1968) « Le mode de tradition des actes écrits et les études en dialectologie ». Revue de linguistique romane $32: 17-47$. 
MONJOUR, A. (1989) « Der Nordostfranzösische Dialektraum ». Bonner romanistische Arbeiten, Band 32. Frankfurt/Bern/New York/Paris : Peter Lang.

MUGGLESTONE, L. (1995) Talking Proper. Oxford : Oxford University Press.

MUSCATINE, C. (1981) «Courtly literature and vulgar language ». In : G.S. Burgess and A. D. Deyermond (dirs), Court and Poet. Liverpool : Francis Cairns, 1-19.

PARIS, G. (1889) « Les parlers de France », Romania 17 : 475-489.

PASSY, P. (1891) « Le patois de Sainte-Jamme (Seine-et-Oise) », Revue des patois gallo-romans 4, 13 : 7-16.

PENNY, R. (2000) Variation and Change in Spanish, Cambridge : Cambridge University Press.

PFISTER, M. (1973) « Die sprachliche Bedeutung von Paris und der Ile-de-France vor dem 13.Jh ». Vox Romanica 32 : 217-253.

PFISTER, M. (1993) « Scripta et koinè en ancien français aux XII ${ }^{\mathrm{e}}$ et XIII ${ }^{\mathrm{e}}$ siècles », in Ecritures, langues communes et normes : formation spontanée de koinès et standardisation dans la Gallo-Romania et son voisinage. Neuchâtel/Genève : Université de Neuchâtel/Droz, 17-41.

PICOCHE, J. et MARCHELLO-NIZIA, C. (1994) Histoire de la langue française (4e éd.), Paris : Nathan. POERCK, G. de (1963) «Les plus anciens textes de la langue française comme témoins d'époque », Revue de linguistique romane 27 : 1-34.

POPE, M.K. (1935) From Latin to Modern French, Manchester : Manchester University Press.

RAUHUT, F. (1963) «Warum wurde Paris die Hauptstadt Frankreichs ? », in H. Bihler and A. Noyer-Weidner (dirs), Medium Aevum Romanicum. Festechrift für E. Reinfelder. Munich : Huber.

REMACLE, L. (1948) Le Problème de l'ancien wallon, Liège : Bibliothèque de la Faculté de Philosophie et Lettres.

RICKARD, P. (1989) A History of the French Language, Londres : Hutchinson.

ROBSON, C.A. (1955) « Literary language, spoken dialect, and the phonological problem in old French », Transactions of the Philological Society 1955 : 117-180

Sarcelades 1731-48. N. Jouin, Le Vrai Recueil des Sarcelles ; mémoires, notes et anecdotes intéressantes sur la conduite de l'archevêque de Paris. Amsterdam 1764.

SAUSSURE, F. de (1971) Cours de linguistique générale, Paris : Payot.

SIEGEL, J. (1985) « Koines and koinéization », Language in Society 14 : 357-378.

SIMONI-AUREMBOU, M.-R. (1973a) Atlas linguistique et ethnographique de l'Ile-de-France et de l'Orléanais (ALIFO). Paris : CNRS.

SIMONI-AUREMBOU, M.-R. (1973b) « Aspects phonétiques de l'atlas de l'Ile-de-France et de l'Orléanais : unité ou diversité ? ", in Les Dialectes romans à la lumière des atlas régionaux. Paris : CNRS, pp.378-96.

SIMONI-AUREMBOU, M.-R. (1995) «L'aire linguistique centrale », in P. Gauthier et T. Lavoie (dirs), Français de France et français du Canada. Lyon : Université Jean Moulin, pp.251-306.

SIMONI-AUREMBOU, M.-R. (1999) « Aperçus sur la langue de l'Ile-de-France », in Chaurand 1999 : 564-572.

TRASK, R.L. (1997) A Student 's Dictionary of Language and Linguistics, Londres : Arnold.

TRUDGILL, P. (1986) Dialects in Contact, Oxford : Blackwell. 
WACKER, G. (1916) Ueber das Verhältnis von Dialekt und Schriftsprache, Halle : Niemeyer.

WALTER, H. (1988) Le Français dans tous les sens, Paris : Laffont.

WARTBURG, W. von (1962) Evolution et structure de la langue française, Berne : Francke.

WEINREICH, U. (1953) Languages in Contact, La Haye : Mouton.

WÜEST, J. (1979a) « Sprachgrenzen im Poitou », Vox Romanica 29 : 14-58.

WÜEST, J. (1979b) La Dialectalisation de la Gallo-Romania, Berne : Francke.

WÜEST, J. (1985) « Le patois de Paris et l'histoire du français », Vox Romanica 44 : 234-58.

\section{AUTEUR}

ANTHONY LODGE

Université de St Andrews (G.B.) 